\title{
Practice of the Incomplete $p$-Ramification Over a Number Field - History of Abelian $p$-Ramification
}

\author{
Georges Gras ${ }^{1 *}$
}

\begin{abstract}
The theory of $p$-ramification, regarding the Galois group of the maximal pro- $p$-extension of a number field $K$, unramified outside $p$ and $\infty$, is well known including numerical experiments with PARI/GP programs. The case of "incomplete $p$-ramification" (i.e., when the set $S$ of ramified places is a strict subset of the set $P$ of the $p$-places) is, on the contrary, mostly unknown in a theoretical point of view. We give, in a first part, a way to compute, for any $S \subseteq P$, the structure of the Galois group of the maximal $S$-ramified abelian pro- $p$-extension $H_{K, S}$ of any field $K$ given by means of an irreducible polynomial. We publish PARI/GP programs usable without any special prerequisites. Then, in an Appendix, we recall the "story" of abelian $S$-ramification restricting ourselves to elementary aspects in order to precise much basic contributions and references, often disregarded, which may be used by specialists of other domains of number theory. Indeed, the torsion $\mathscr{T}_{K, S}$ of $\operatorname{Gal}\left(H_{K, S} / K\right.$ ) (even if $S=P$ ) is a fundamental obstruction in many problems. All relationships involving $S$-ramification, as Iwasawa's theory, Galois cohomology, $p$-adic $L$-functions, elliptic curves, algebraic geometry, would merit special developments, which is not the purpose of this text.
\end{abstract}

Keywords: Abelian $S$-ramification, Class field theory, Class groups, Leopoldt's conjecture, $p$-adic regulators, Pro- $p$-groups, Units, $\mathbb{Z}_{p}$-extensions

2010 AMS: 11R37, 11F85, 11R34, 11Y40, 08-04

${ }^{1}$ Villa la Gardette, chemin Château Gagnière, F-38520 Le Bourg d'Oisans, ORCID: 0000-0002-1318-4414

*Corresponding author g.mn.gras@wanadoo.fr

Received: 04 June 2019, Accepted: 19 September 2019, Available online: 29 December 2019

\section{Introduction and basic results}

The numerous references that we mention allow the reader to have a chronological overview of the contributions. Many results have been collected in our book (Edit. 2005) quoted [1].

\subsection{Notion of Galois $S$-ramification}

Let $p \geq 2$ be a prime number and let $K$ be a number field; we denote by $P:=\{\mathfrak{p}$ prime, $\mathfrak{p} \mid p\}$ the set of $p$-places of $K$ and by $S$ an arbitrary set of finite places (later we shall assume $S \subseteq P$ ).

A main problem in Galois theory above $K$ is to study the Galois group $\mathscr{G}_{K, S}$ of the maximal pro-p-extension of $K$ which is $S$-ramified in the ordinary sense (i.e., unramified outside $S$ and non-complexified (= totally split) at the real infinite places of $K$ when $p=2$ ).

As we will recall it in detail, in Section A.2, the study of $\mathscr{G}_{K, S}$ goes back to fundamental contributions of Serre [2], Šafarevič [3], Brumer [4], and has been largely extended, from the 1980's, in much works considering $S$-ramification (eventually with decomposition of another set $\Sigma$ of finite and infinite places). 
The analogous theory for a local base field has also a long history that we shall not consider in this article.

\subsection{Main cohomological invariants}

For complete current information about the "cohomology of number fields", see the book of Neukirch-Schmidt-Wingberg [5, Chapter X].

When $S=P$, the $\mathbb{F}_{p}$-dimension of $\mathrm{H}^{1}\left(\mathscr{G}_{K, P}, \mathbb{Z} / p \mathbb{Z}\right)$, which gives the minimal number of generators of $\mathscr{G}_{K, P}$, is the $p$-rank ${ }^{1}$ of the abelianization:

$$
\mathscr{A}_{K, P}:=\mathscr{G}_{K, P}^{\mathrm{ab}}:=\mathscr{G}_{K, P} /\left[\mathscr{G}_{K, P}, \mathscr{G}_{K, P}\right] .
$$

Denote by $\left(r_{1}, r_{2}\right)$ the signature of $K$ (whence $\left.r_{1}+2 r_{2}=[K: \mathbb{Q}]\right)$; then, the $\mathbb{F}_{p}$-dimension of $\mathrm{H}^{2}\left(\mathscr{G}_{K, P}, \mathbb{Z} / p \mathbb{Z}\right)$, which gives the minimal number of relations between these generators, fulfills the identity:

$$
\operatorname{rk}_{p}\left(\mathrm{H}^{1}\left(\mathscr{G}_{K, P}, \mathbb{Z} / p \mathbb{Z}\right)\right)=\operatorname{rk}_{p}\left(\mathrm{H}^{2}\left(\mathscr{G}_{K, P}, \mathbb{Z} / p \mathbb{Z}\right)\right)+r_{2}+1,
$$

giving, for the torsion group $\mathscr{T}_{K, P}$ of $\mathscr{A}_{K, P}$ under Leopoldt's conjecture:

$$
\operatorname{rk}_{p}\left(\mathscr{T}_{K, P}\right)=\operatorname{rk}_{p}\left(\mathrm{H}^{2}\left(\mathscr{G}_{K, P}, \mathbb{Z} / p \mathbb{Z}\right)\right) .
$$

\subsection{Class field theory}

In the general case for $S$ (possibly containing tame places and not all the $p$-places) we may write:

$$
\mathscr{A}_{K, S}=\Gamma_{K, S} \oplus \mathscr{T}_{K, S}, \text { with } \Gamma_{K, S} \simeq \mathbb{Z}_{p}^{\widetilde{r}_{K, S}},
$$

where $\mathscr{T}_{K, S}:=\operatorname{tor}_{\mathbb{Z}_{p}}\left(\mathscr{A}_{K, S}\right)$ and $\widetilde{r}_{K, S} \geq 0$.

Without any $p$-adic assumption on the group of global units of $K$, we still have $\operatorname{rk}_{p}\left(\mathrm{H}^{1}\left(\mathscr{G}_{K, S}, \mathbb{Z} / p \mathbb{Z}\right)\right)=\operatorname{rk}_{p}\left(\mathscr{A}_{K, S}\right)$, but $\widetilde{r}_{K, S}$ (called the $\mathbb{Z}_{p}$-rank of $\left.\mathscr{A}_{K, S}\right)$ is more difficult when $S \subsetneq P$; however, $\operatorname{rk}_{p}\left(\mathscr{A}_{K, S}\right)=\widetilde{r}_{K, S}+\operatorname{rk}_{p}\left(\mathscr{T}_{K, S}\right)$ is computable in complete generality with the invariants of class field theory for $K$ as follows (Šafarevič formula):

Let $K_{(S)}^{\times}$be the subgroup of $K^{\times}$of elements prime to $S$ and for any $\mathfrak{p} \in S$, let $K_{\mathfrak{p}}$ be the completion of $K$ at $\mathfrak{p}$; then:

$$
\operatorname{rk}_{p}\left(\mathscr{A}_{K, S}\right)=\operatorname{rk}_{p}\left(V_{K, S} / K_{(S)}^{\times p}\right)+\sum_{\mathfrak{p} \in S \cap P}\left[K_{\mathfrak{p}}: \mathbb{Q}_{p}\right]+\sum_{\mathfrak{p} \in S} \delta_{\mathfrak{p}}-\delta_{K}-\left(r_{1}+r_{2}-1\right),
$$

where $V_{K, S}:=\left\{\alpha \in K_{(S)}^{\times},(\alpha)=\mathfrak{a}^{p}\right.$ for an ideal $\mathfrak{a}$ of $\left.K\right\}, \delta_{\mathfrak{p}}=1$ or 0 according as $K_{\mathfrak{p}}$ contains $\mu_{p}$ or not, and $\delta_{K}=1$ or 0 according as $K$ contains $\mu_{p}$ or not. Thus, from the relation (1.1):

$$
\operatorname{rk}_{p}\left(\mathscr{T}_{K, S}\right)=\operatorname{rk}_{p}\left(\mathscr{A}_{K, S}\right)-\widetilde{r}_{K, S}=\operatorname{rk}_{p}\left(V_{K, S} / K_{(S)}^{\times p}\right)+\left[\sum_{\mathfrak{p} \in S \cap P}\left[K_{\mathfrak{p}}: \mathbb{Q}_{p}\right]-\widetilde{r}_{K, S}\right]+\sum_{\mathfrak{p} \in S} \delta_{\mathfrak{p}}-\delta_{K}-\left(r_{1}+r_{2}-1\right),
$$

where $\widetilde{r}_{K, S}$ fulfills the following formula:

$$
\sum_{\mathfrak{p} \in S \cap P}\left[K_{\mathfrak{p}}: \mathbb{Q}_{p}\right]-\widetilde{r}_{K, S}=\operatorname{dim}_{\mathbb{Q}_{p}}\left(\mathbb{Q}_{p} \log _{S \cap P}\left(E_{K}\right)\right),
$$

where $E_{K}$ is the group of global units of $K$ and $\log _{S \cap P}:=\left(\log _{\mathfrak{p}}\right)_{\mathfrak{p} \in S \cap P}$ the family of $p$-adic logarithms over $S \cap P$ with values in $\bigoplus_{\mathfrak{p} \in S \cap P} K_{\mathfrak{p}}$. Note that for $S=P$,

$$
r_{K, P}:=\operatorname{dim}_{\mathbb{Q}_{p}}\left(\mathbb{Q}_{p} \log _{P}\left(E_{K}\right)\right),
$$

is the $p$-adic rank of $E_{K}$ (i.e., the $\mathbb{Z}_{p}$-rank of the closure of the image $\imath_{P}\left(E_{K}\right)$ of $E_{K}$ in the group of local principal units $U_{K, P}$, where $l_{P}$ is the diagonal embedding; see $\S 2.1$ ).

The Šafarevič and reflection formulas, generalized with decomposition, may be obtained via [1, Exercise II.5.4.1] or other classical references.

In general, $\widetilde{r}_{K, S}$ is non-obvious and varies from 0 to $r_{2}+1$ (see Wingberg [6, 7], Yamagishi [8], Maire [9, 10, 11], Labute [12], [13], Vogel [14] for some results and cases where $\mathscr{G}_{K, S}$ may be free with less than $r_{2}+1$ generators and our forthcoming numerical results showing that many $\mathbb{Z}_{p}$-ranks can occur).

\footnotetext{
${ }^{1}$ As usual, the $p$-rank of an abelian group $A$ is the $\mathbb{F}_{p}$-dimension of $A / A^{p}$.
} 
For $S=P$ we obtain $\widetilde{r}_{K, P}=r_{2}+1$, under the Leopoldt conjecture, giving (since $\left.\sum_{\mathfrak{p} \in P}\left[K_{\mathfrak{p}}: \mathbb{Q}_{p}\right]=r_{1}+2 r_{2}\right)$ :

$$
\operatorname{rk}_{p}\left(\mathscr{T}_{K, P}\right)=\operatorname{rk}_{p}\left(V_{K, P} / K_{P}^{\times p}\right)+\sum_{\mathfrak{p} \in P} \delta_{\mathfrak{p}}-\delta_{K}
$$

If $S=\emptyset$ then $\mathscr{A}_{K, S}=\mathscr{T}_{K, S}=: C \ell_{K}$, the $p$-class group of $K$ (ordinary sense).

Remark 1.1. We shall not consider $S$-ramification with $S=P \cup T$, when $T$ is a finite set of tame places, because of the following exact sequence, under the Leopoldt conjecture (Neumann [15], Nguyen Quang Do [16, Corollary 4.3], [1, Theorem III.4.1.5]), where the $F_{\mathfrak{l}}$ are the residue fields:

$$
1 \longrightarrow \bigoplus_{\mathfrak{l} \in T}\left(F_{\mathfrak{l}}^{\times} \otimes \mathbb{Z}_{p}\right) \longrightarrow \mathscr{T}_{K, P \cup T} \longrightarrow \mathscr{T}_{K, P} \longrightarrow 1
$$

For some specialized applications (about number fields, elliptic curves, representation theory, Galois cohomology, Iwasawa's theory, $p$-adic $L$-functions) and some recent conjectures, one needs to study and compute the above $S$-invariants when $S$ is a subset of $P$ and $K / \mathbb{Q}$ not necessarily Galois. Even if $K / \mathbb{Q}$ is Galois, the Galois group does not necessarily operate on $S$. So the classical algebraic considerations (cohomology, Iwasawa's theory) largely collapse.

So the most tricky invariants in "incomplete $P$-ramification" are

$$
\mathscr{T}_{K, S} \text { and } \widetilde{r}_{K, S}=\operatorname{rk}_{p}\left(\mathscr{A}_{K, S}\right)-\operatorname{rk}_{p}\left(\mathscr{T}_{K, S}\right)=\sum_{\mathfrak{p} \in S}\left[K_{\mathfrak{p}}: \mathbb{Q}_{p}\right]-\operatorname{dim}_{\mathbb{Q}_{p}}\left(\mathbb{Q}_{p} \log _{S}\left(E_{K}\right)\right)
$$

Of course, they highly depend on the decomposition of the prime $p$ in the Galois closure of $K$ and probably of specific $p$-adic properties of units; but it remains the class field theory framework above the base field $K$.

\section{General $p$-adic context of $S$-ramification}

Consider a number field $K$ and a given prime $p \geq 2$. Let $S$ be a subset of the set $P$ of the $p$-places of $K$ and let $H_{K, S}$ be the maximal abelian $S$-ramified pro- $p$-extension of $K$; this field contains a (maximal) compositum $\widetilde{K}^{S}$ of $\mathbb{Z}_{p}$-extensions of $K$ and always the $p$-Hilbert class field $H_{K}:=H_{K, \emptyset}$ of $K$.

These definitions are given in the ordinary sense when $p=2$ (so that the real infinite places of $K$ are not complexified in the class fields considered; in other words they are totally split).

\subsection{Fundamental exact sequences}

Let $U_{K, S}:=\bigoplus_{\mathfrak{p} \in S} U_{\mathfrak{p}}$, be the product of the groups of principal local units of $K_{\mathfrak{p}}, \mathfrak{p} \in S$, and let $\bar{E}_{K}^{S}$ be the closure of the image $\imath_{S}\left(E_{K}\right)$ of $E_{K}$ in $U_{K, S}$.

We denote by $W_{K, S}=\bigoplus_{\mathfrak{p} \in S} \mu_{K_{\mathfrak{p}}}$ the torsion group of the $\mathbb{Z}_{p}$-module $U_{K, S}$.

If $K / \mathbb{Q}$ is Galois and $S \subsetneq P, U_{K, S}$ is not necessarily a Galois module, which increases the difficulties.

The following $p$-adic result is valid without any assumption on $K$ and $S \subseteq P$ :

Lemma 2.1. We have the exact sequence:

$$
1 \rightarrow W_{K, S} / \operatorname{tor}_{\mathbb{Z}_{p}}\left(\bar{E}_{K}^{S}\right) \longrightarrow \operatorname{tor}_{\mathbb{Z}_{p}}\left(U_{K, S} / \bar{E}_{K}^{S}\right) \stackrel{\log _{S}}{\longrightarrow} \operatorname{tor}_{\mathbb{Z}_{p}}\left(\log _{S}\left(U_{K, S}\right) / \log _{S}\left(\bar{E}_{K}^{S}\right)\right) \rightarrow 0 .
$$

Proof. The surjectivity comes from the fact that if $u \in U_{K, S}$ is such that $p^{n} \log _{S}(u)=\log _{S}(\bar{\varepsilon}), \bar{\varepsilon} \in \bar{E}_{K}^{S}$, then $u^{p^{n}}=\bar{\varepsilon} \cdot \xi$ for $\xi \in W_{K, S}$, hence there exists $m \geq n$ such that $u^{p^{m}} \in \bar{E}_{K}^{S}$, whence $u$ gives a preimage in tor $_{\mathbb{Z}_{p}}\left(U_{K, S} / \bar{E}_{K}^{S}\right)$. If $u \in U_{K, S}$ is such that $\log _{S}(u) \in \log _{S}\left(\bar{E}_{K}^{S}\right)$, then $u=\bar{\varepsilon} \cdot \xi$ as above, giving the kernel equal to $\bar{E}_{K}^{S} \cdot W_{K, S} / \bar{E}_{K}^{S}=W_{K, S} / \operatorname{tor}_{\mathbb{Z}_{p}}\left(\bar{E}_{K}^{S}\right)$.

Put:

$$
\mathscr{W}_{K, S}:=W_{K, S} / \operatorname{tor}_{\mathbb{Z}_{p}}\left(\bar{E}_{K}^{S}\right) \quad \& \quad \mathscr{R}_{K, S}:=\operatorname{tor}_{\mathbb{Z}_{p}}\left(\log _{S}\left(U_{K, S}\right) / \log _{S}\left(\bar{E}_{K}^{S}\right)\right) .
$$

Then the exact sequence of Lemma 2.1 becomes:

$$
1 \longrightarrow \mathscr{W}_{K, S} \longrightarrow \operatorname{tor}_{\mathbb{Z}_{p}}\left(U_{K, S} / \bar{E}_{K}^{S}\right) \stackrel{\log _{S}}{\longrightarrow} \mathscr{R}_{K, S} \longrightarrow 0
$$


Lemma 2.2. Let $\mu_{K}$ be the group of roots of unity of p-power order of $K$. Under the Leopoldt conjecture for $p$ in $K$ we have tor $_{\mathbb{Z}_{p}}\left(\bar{E}_{K}^{P}\right)=\imath_{P}\left(\mu_{K}\right)$; thus, in that case, $\mathscr{W}_{K, P}=W_{K, P} / \imath_{P}\left(\mu_{K}\right)$.

Proof. From Jaulent [17, Définition 2.11, Proposition 2.12] or [1, Theorem III.3.6.2(vi)].

Note that for $S \subsetneq P$, we do not know if $\operatorname{tor}_{\mathbb{Z}_{p}}\left(\bar{E}_{K}^{S}\right)$ may be larger than $\imath_{S}\left(\mu_{K}\right)$ (as subgroups of $W_{K, S}$ ), even under the Leopoldt conjecture.

\subsection{Diagram of $S$-ramification}

Consider the following diagram under the Leopoldt conjecture for $p$ in $K$. By definition, $\mathscr{T}_{K, S}=$ tor $_{\mathbb{Z}_{p}}\left(\mathscr{A}_{K, S}\right)$ is the Galois group $\operatorname{Gal}\left(H_{K, S} / \widetilde{K}^{S}\right)$; let $\widetilde{C \ell_{K}}$ be the subgroup of $C \ell_{K}$ corresponding to $\operatorname{Gal}\left(H_{K} / \widetilde{K}^{S} \cap H_{K}\right)$ by class field theory.

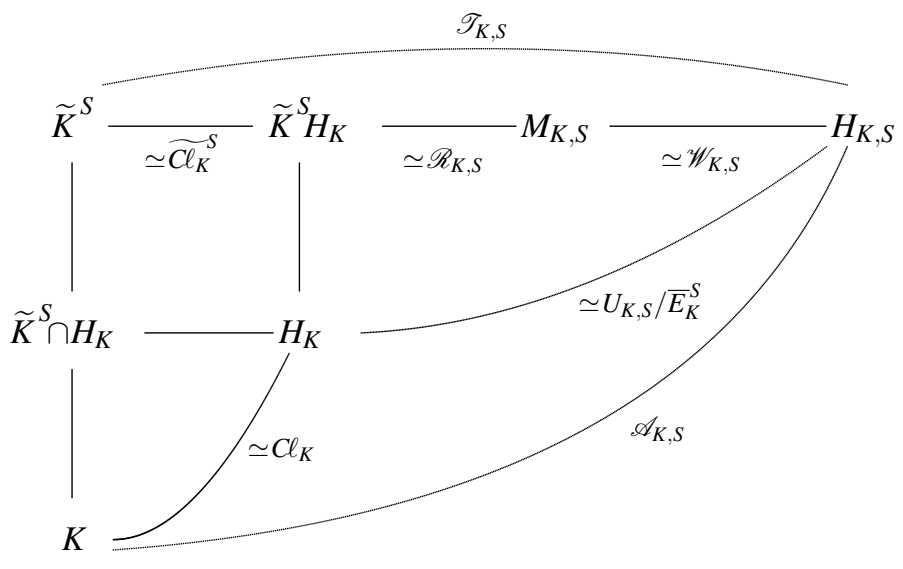

Then from the schema we get:

$$
\# \mathscr{T}_{K, S}=\left[H_{K}: \widetilde{K}^{S} \cap H_{K}\right] \cdot \# \operatorname{tor}_{\mathbb{Z}_{p}}\left(U_{K, S} / \bar{E}_{K}^{S}\right)={\widetilde{C \ell_{K}}}^{S} \cdot \# \mathscr{R}_{K, S} \cdot \# \mathscr{W}_{K, S}
$$

Of course, for $p \geq p_{0}$ (explicit), $\# \mathscr{W}_{K, S}={\widetilde{C \ell_{K}}}^{S}=1$, whence $\mathscr{T}_{K, S}=\mathscr{R}_{K, S}$.

Remark 2.3. When $S=P$, we have $\operatorname{Gal}\left(H_{K, P} / H_{K}\right) \simeq U_{K, P} / \bar{E}_{K}^{P}$, in which the image of $\mathscr{W}_{K, P}$ fixes $M_{K, P}=: H_{K}^{\mathrm{bp}}$, the BertrandiasPayan field, $\mathrm{Gal}\left(H_{K}^{\mathrm{bp}} / \widetilde{K}^{P}\right)$ being the Bertrandias-Payan module as named by Nguyen Quang Do from [18] on the p-cyclic embedding problem. Then $\mathscr{R}_{K, P} \simeq \operatorname{Gal}\left(H_{K}^{\mathrm{bp}} / \widetilde{K}^{P} H_{K}\right)$. This “normalized regulator" $\mathscr{R}_{K, P}$ (as a p-group or as a p-power) is closely related to the classical p-adic regulator of $K$ (see [19, Proposition 5.2]).

\subsection{Local computations}

Recall the following local computation:

Theorem 2.4. [1, Theorem I.4.5 \& Corollary I.4.5.4, ordinary sense]. For $\mathfrak{p} \mid p$ in $K$ and $j \geq 1$, let $U_{\mathfrak{p}}^{j}$ be the group of local units $1+\overline{\mathfrak{p}}^{j}$, where $\overline{\mathfrak{p}}$ is the maximal ideal of the ring of integers of $K_{\mathfrak{p}}$. For $S \subseteq P$, denote by $\mathfrak{m}(S)$ the modulus $\prod_{\mathfrak{p} \in S} \mathfrak{p}^{e_{\mathfrak{p}}}$, where $e_{\mathfrak{p}}$ is the ramification index of $\mathfrak{p}$ in $K / \mathbb{Q}$.

For a modulus of the form $\mathfrak{m}(S)^{n}, n \geq 0$, let $C \ell_{K}\left(\mathfrak{m}(S)^{n}\right)$ be the corresponding ray class group (ordinary sense). Then for $m \geq n \geq 0$, we have:

$$
0 \leq \operatorname{rk}_{p}\left(C \ell_{K}\left(\mathfrak{m}(S)^{m}\right)\right)-\operatorname{rk}_{p}\left(C \ell_{K}\left(\mathfrak{m}(S)^{n}\right)\right) \leq \sum_{\mathfrak{p} \in S} \operatorname{rk}_{p}\left(\left(U_{\mathfrak{p}}^{1}\right)^{p} U_{\mathfrak{p}}^{n \cdot e_{\mathfrak{p}}} /\left(U_{\mathfrak{p}}^{1}\right)^{p} U_{\mathfrak{p}}^{m \cdot e_{\mathfrak{p}}}\right)
$$

Corollary 2.5. [20, Theorem 2.1 \& Corollary 2.2] We have:

$$
\operatorname{rk}_{p}\left(C \ell_{K}\left(\mathfrak{m}(S)^{m}\right)\right)=\operatorname{rk}_{p}\left(C \ell_{K}\left(\mathfrak{m}(S)^{n}\right)\right)=\operatorname{rk}_{p}\left(\mathscr{A}_{K, S}\right), \text { for all } m \geq n \geq n_{0},
$$

where $n_{0}=3$ for $p=2$ and $n_{0}=2$ for $p>2$. Thus $\mathscr{T}_{K, S}=1$ if and only if $\operatorname{rk}_{p}\left(C \ell_{K}\left(\mathfrak{m}(S)^{n_{0}}\right)\right)=\widetilde{r}_{K, S}\left(\mathbb{Z}_{p}\right.$-rank of $\left.\mathscr{A}_{K, S}\right)$. 
Proof. It is sufficient to get, for some fixed $n \geq 0$ :

$$
\left(U_{\mathfrak{p}}^{1}\right)^{p} U_{\mathfrak{p}}^{n \cdot e_{\mathfrak{p}}}=\left(U_{\mathfrak{p}}^{1}\right)^{p}, \text { for all } \mathfrak{p} \in S,
$$

hence $U_{\mathfrak{p}}^{n \cdot e_{\mathfrak{p}}} \subseteq\left(U_{\mathfrak{p}}^{1}\right)^{p}$ for all $\mathfrak{p} \in S$; indeed, we then have:

$$
\operatorname{rk}_{p}\left(C \ell_{K}\left(\mathfrak{m}(S)^{n}\right)\right)=\operatorname{rk}_{p}\left(C \ell_{K}\left(\mathfrak{m}(S)^{m}\right)\right)=\widetilde{r}_{K, S}+\operatorname{rk}_{p}\left(\mathscr{T}_{K, S}\right) \text { as } m \rightarrow \infty
$$

giving $\operatorname{rk}_{p}\left(C \ell_{K}\left(\mathfrak{m}(S)^{n}\right)\right)=\widetilde{r}_{K, S}+\operatorname{rk}_{p}\left(\mathscr{T}_{K, S}\right)$ for such $n$.

The condition $U_{\mathfrak{p}}^{n \cdot e_{\mathfrak{p}}} \subseteq\left(U_{\mathfrak{p}}^{1}\right)^{p}$ is fulfilled as soon as $n \cdot e_{\mathfrak{p}}>\frac{p \cdot e_{\mathfrak{p}}}{p-1}$, whence $n>\frac{p}{p-1}$ (Fesenko-Vostokov [21, Chapter I, $\S 5.8$, Corollary 2]) giving the value of $n_{0}$; furthermore, $C \ell_{K}\left(\mathfrak{m}(S)^{n_{0}}\right)$ gives the $p$-rank of $\mathscr{T}_{K, S}$ as soon as the $\mathbb{Z}_{p}$-rank $\widetilde{r}_{K, S}$ is known.

\subsection{Practical computation of $\widetilde{r}_{K, S}$}

Let $S \subseteq P$. From (1.3), we have: $\widetilde{r}_{K, S}=\sum_{\mathfrak{p} \in S}\left[K_{\mathfrak{p}}: \mathbb{Q}_{p}\right]-r_{K, S}$, where $r_{K, S}:=\operatorname{dim}_{\mathbb{Q}_{p}}\left(\mathbb{Q}_{p} \log _{S}\left(E_{K}\right)\right)$.

(i) In $[9,10]$ Maire has given, in the relative Galois case, some results about $r_{K, S}$ depending on Schanuel's conjecture and the use of the representation $\mathbb{Q}_{p} \log _{S}\left(E_{K}\right)$ from the results of Jaulent [22].

(ii) In the Galois case, this rank has been studied by Nelson [23] giving formulas (or lower bounds) under the $p$-adic Schanuel conjecture.

(iii) We have proposed, in [1, III, $\S 4$ (f)], a conjecture and a calculation process in the general non-Galois case using a Galois descent from the Galois closure $N$ of $K$ and the family of decomposition groups of the places of $N$ above $p$ and $\infty$. If $K / \mathbb{Q}$ is Galois then (with $\Sigma:=P \backslash S$ ):

$$
\operatorname{rk}_{\mathbb{Z}_{p}}\left(\operatorname{Gal}\left(\widetilde{K}^{P} / \widetilde{K}^{S}\right)\right)=\sum_{\mathfrak{p} \in \Sigma}\left[K_{\mathfrak{p}}: \mathbb{Q}_{p}\right]-\operatorname{dim}_{\mathbb{Q}_{p}}\left(\mathbb{Q}_{p} \log _{P}\left(\mathscr{E}_{K, S}\right)\right)
$$

where:

$$
\mathscr{E}_{K, S}:=\left\{\varepsilon \in E_{K} \otimes \mathbb{Z}_{p}, \quad l_{\mathfrak{p}}(\varepsilon)=1, \forall \mathfrak{p} \in S\right\} \quad \& \quad l_{\mathfrak{p}}: E_{K} \otimes \mathbb{Z}_{p} \rightarrow U_{\mathfrak{p}}^{1}
$$

But all these similar approaches are difficult for programming and not so obvious for random $K$ and $S$ because of conjectural aspects; so we shall preferably give extensive computations via PARI/GP [24] since ray class fields are well computed. But it remains the problem of justification of the "computing" of $\widetilde{r}_{K, S}$, when no theoretical value is known (see another explicit numerical method in $[1, \S$ III.5, Theorem 5.2]).

We conclude by the following comments:

Remark 2.6. If $\mathscr{T}_{K, P}=1$ (i.e., the field $K$ is called p-rational as proposed by Movahhedi in [25, 26]), this does not imply $\mathscr{T}_{K, S}=1$ for $S \subsetneq P$ (the numerical examples will show many cases). In the opposite situation, we may have $\mathscr{T}_{K, P} \neq 1$, but often $\mathscr{T}_{K, S}=1$ for $S \subsetneq P$.

This intricate aspects have been studied by Maire [11, Section 3] in which he introduces the "S-cohomologcal condition" $\mathrm{H}^{2}\left(\mathscr{G}_{K, S}, \mathbb{Q}_{p} / \mathbb{Z}_{p}\right)=0$ (knowing that $\mathscr{G}_{K, S}$ is a free prop-group if and only if $\mathrm{H}^{2}\left(\mathscr{G}_{K, S}, \mathbb{Q}_{p} / \mathbb{Z}_{p}\right)$ and $\mathscr{T}_{K, S}$ are trivial) and that of "S-arithmetical condition" ( $E_{K} \otimes \mathbb{Z}_{p} \rightarrow U_{K, S}$ injective), and compare them, which of course coincide for $S=P$; we know that the $S$-arithmetical condition implies the $S$-cohomologcal one.

We shall speak of $S$-rationality, when $\mathscr{T}_{K, S}=1$ for $S \subseteq P$, even if this may be rather ambiguous when $S \subsetneq P$ because of the above observations; one must understand this as a "free $S$-ramification" over $K$ (i.e., giving a free abelian S-ramified pro-p-extension $\left.H_{K, S} / K\right)$. This is also justified by the fact that many variants of the definition have been given, as those of Jaulent-Sauzet [27, 28], Bourbon-Jaulent [29], where are defined and studied the case of singleton $S=\{\mathfrak{p}\}$ or that of the "2-birationality" of quadratic extensions of totally real fields when $S=\left\{\mathfrak{p}, \mathfrak{p}^{\prime}\right\}$.

\section{Algorithmic approach of $S$-ramification}

The principle is to consider a modulus $\mathfrak{m}_{S}:=\prod_{\mathfrak{p} \in S} \mathfrak{p}^{\lambda_{\mathfrak{p}}}, S \subseteq P$, with $\lambda_{\mathfrak{p}} \gg 0$ for all $\mathfrak{p} \in S$ to "read" the structure of $\mathscr{A}_{K, S}$ on the ray class group $C \ell_{K}\left(\mathfrak{m}_{S}\right)$. The practice shows that the more convenient modulus is of the form:

$$
\left(\prod_{\mathfrak{p} \in S} \mathfrak{p}^{e_{\mathfrak{p}}}\right)^{n},
$$

where $e_{\mathfrak{p}}$ is the ramification index of $\mathfrak{p}$ in $K / \mathbb{Q}$ and $n \gg 0$. Of course, this modulus is $\left(p^{n}\right)$ only for $S=P$; so we must use the ideal decomposition of $p$ in $K$, given by PARI/GP, and compute everywhere with ideals. 


\subsection{Main program computing $\mathscr{T}_{K, S}$ and $\widetilde{r}_{K, S}$}

\subsubsection{The PARI/GP program}

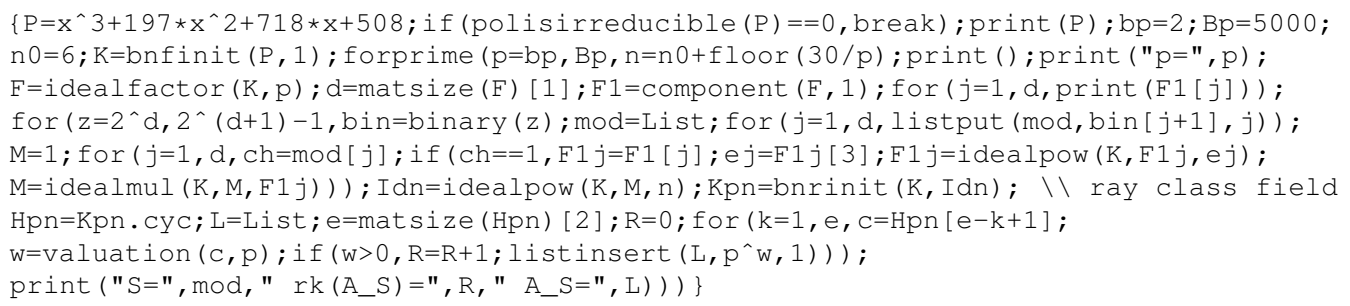

\subsubsection{Instructions for use and illustrations}

See the Note at the end of Section A.8. The reader has only to copy and past the verbatim of the program and to use a "terminal session via Sage", on his or her computer, or a cell in the page http://pari.math.u-bordeaux.fr/gp.html

The programs in this article can be directly copied and pasted at:

https: / / www.dropbox.com/s/1srmksbr2ujf40i/Incomplete\%20p-ramification.pdf?dl=0

It is assumed that the irreducible monic polynomial $\mathrm{P}$ defining $K$ is given and that the interval [bp, Bp] of tested primes $p$ is also given by the user.

(i) The program computes the decomposition of $p$ into $\mathrm{d}$ prime ideals; for instance, the following data gives, for $\mathrm{P}=\mathrm{x}^{3}+197 * \mathrm{x}^{2}+718 * \mathrm{x}+508$ and $p=2$, the decomposition $(p)=\mathfrak{p p}^{\prime}$ in $\mathbb{Q}(x)$, using idealfactor $(\mathrm{K}, \mathrm{p})$ :

$[2,[-65,0,1] \sim, 1,1,[0,0,-1] \sim]$

$[2,[0,0,1] \sim, 1,2,[0,1,0] \sim]$

Recall that for an ideal as $\left[2,[0,0,1]^{\sim}, 1,2,[0,1,0]^{\sim}\right]$, the 3 th component is its ramification index, the 4th component is its residue degree. For the computation of the modulus $\mathfrak{m}_{S}$ (to be considered at the power $n$ ), we replace each prime ideal $\mathfrak{p} \in S$ by $\mathfrak{p}^{e_{\mathfrak{p}}}$ using the function idealpow.

(ii) For each modulus $\mathfrak{m}_{S}=\prod_{\mathfrak{p} \in S} \mathfrak{p}^{e_{\mathfrak{p}} \cdot n}$, the program gives $\operatorname{rk}_{p}\left(\mathscr{A}_{K, S}\right)$ and the $\mathbb{Z}$-structure of $\mathscr{A}_{K, S} / \mathscr{A}_{K, S}^{p^{N}}$, for $N$ of the order of $n$, under the form:

$$
\mathscr{A}_{K, S}=\left[a_{1}, \ldots, a_{r} ; b_{1}, \ldots, b_{t}\right]
$$

where the coefficients $a_{1}, \ldots, a_{r}$ increase (resp. the coefficients $b_{1}, \ldots, b_{t}$ stabilize) as the exponent $n$ increses, so in the non-ambiguous cases, $b_{1}, \ldots, b_{t}$ give the group-invariants of $\mathscr{T}_{K, S}$ and $r$ is the $p$-rank $\widetilde{r}_{K, S}$ of $\operatorname{Gal}\left(\widetilde{K}^{S} / K\right)$.

Of course, if the rank $\widetilde{r}_{K, S}$ is not certain, we can not, in a mathematical point of view, deduce the structure of $\mathscr{T}_{K, S}$; but in practice the information is correct since one can always verify, with the program, the stabilization of the invariants $b_{j}$ whereas the $a_{i}$ increase linearly to infinity.

(iii) The symbolic data $S=\left[\delta_{1}, \ldots, \delta_{d}\right], \delta_{i} \in\{0,1\}$, indicates that the $S$-modulus considered is:

$$
\mathfrak{m}_{S}=\left(\prod_{i=1}^{d} \mathfrak{p}_{i} e_{\mathfrak{p}_{i}} \cdot \delta_{i}\right)^{n}
$$

We have chosen $\mathrm{n}=$ floor $\left(\mathrm{n}_{0}+\frac{30}{\mathrm{p}}\right)$ to get small values when $p \gg 0$ but larger ones for small $p$ (especially $p=2$ giving possibly huge $\# \mathscr{T}_{K, S}$ ). The parameter $n_{0}$ may be increased at will (here $n_{0}=6$ ).

There are $2^{\# S}$ distinct sets $S$ parametrized with the binary writing of the integers $z \in\left[0,2^{d}-1\right]$.

For $S=[0, \ldots, 0]$ one obtains the structure of the $p$-class group $C \ell_{K}$.

(iv) We illustrate the program with an example where $K$ (a totally real cubic field) is not $S$-rational for some small $p$ and some $S \subseteq P$; but in almost all cases, $K$ is $S$-rational.

Remark 3.1. We do not compute the Galois group associated to the given polynomial, nor the discriminant or the fundamental units; otherwise, the reader has only to add if necessary the instructions:

print("Galois :",polgalois(P));

print ("Discriminant: ", factor(component (component $(K, 7), 3))$ );

print ("Fundamental system of units: ", component (component $(K, 8), 5)$ ); 
giving, for the Galois group and the discriminant:

Galoisgroup $=\left[6,-1,1\right.$, ,S3"] in the PARI/GP notation ${ }^{2}$ and Discriminant $\left.=[769,1 ; 1390573,1]\right)$.

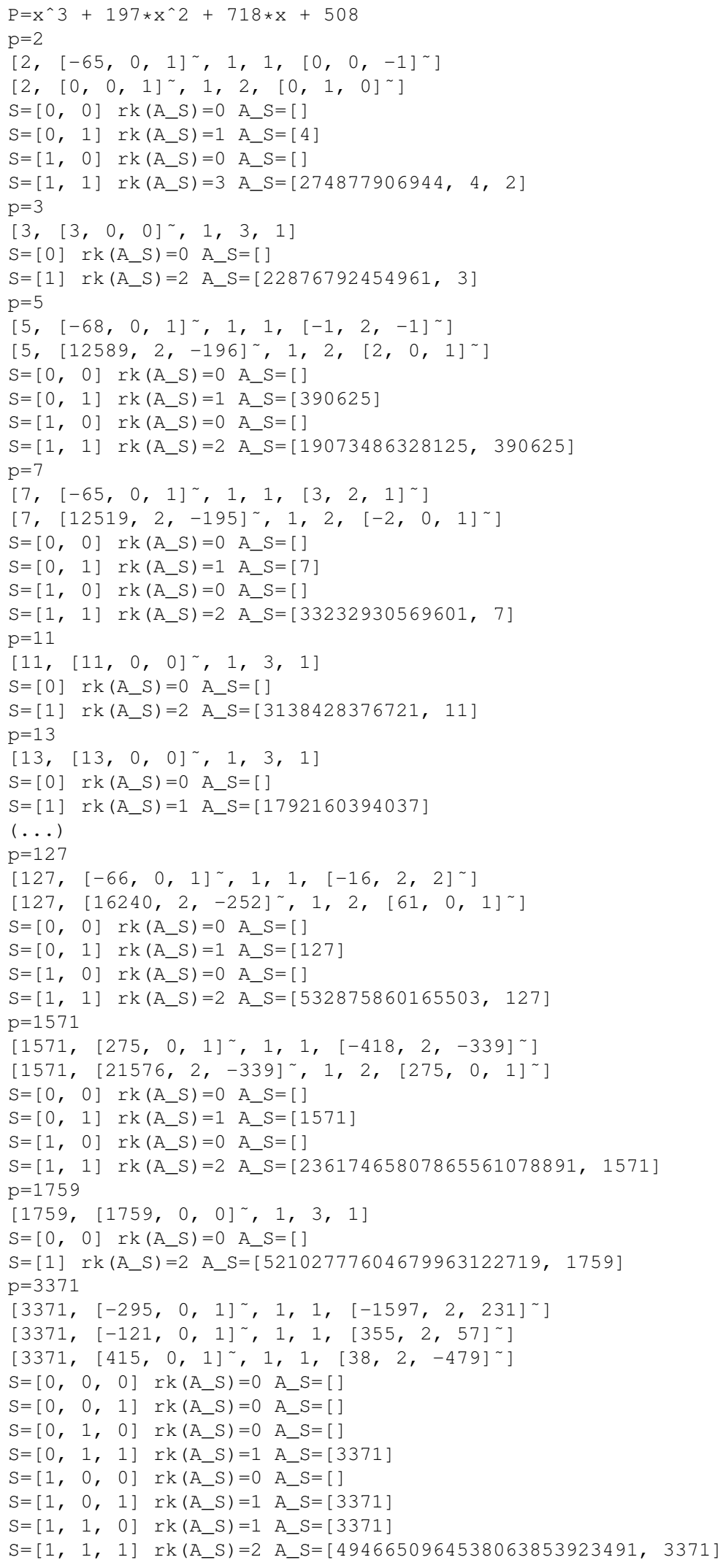

${ }^{2}$ See: http://galoisdb.math. upb.de/home 
If, for the remarquable case $p=5$, one has some doubt, one increases $n$, which gives (for $n=50$ ):

$[5,[-68,0,1] \sim, 1,1,[-1,2,-1] \sim]$

$[5,[12589,2,-196] \sim, 1,2,[2,0,1] \sim]$

$S=[0,0] \quad r k($ A_S $)=0$ A_S $=[]$

$S=[0,1] \quad r k($ A_S $)=1$ A_S $=[390625]$

$S=[1,0] \quad r k($ A_S $)=0$ A_S $=[]$

$S=[1,1]$ rk (A_S) $=2$ A_S $=[17763568394002504646778106689453125 ， 390625]$

Whence $\mathscr{T}_{K, S} \simeq \mathbb{Z} / 5^{8} \mathbb{Z}$ for $S_{1}=\{\mathfrak{p}\}$ (for the prime of residue degree 2 ) and $S_{2}=P$. Note that once the substantial computation of $\mathrm{K}=\operatorname{bnfinit}(\mathrm{P}, 1)$ (giving all the basic information about the field) is done, very large values of $n$ do not increase much the execution time; so any skeptical user can make $n \rightarrow \infty$ to see that only the data 390625 remains constant.

(v) In $[30, \S 9.1]$ we have used some special families of polynomials (e.g., Lecacheux-Washington ones) in which we can force the $p$-adic regulator to be $p$-adicaly close to 0 at will; but we must take the parameter $n$ in proportion, even if here the $\mathbb{Z}_{p}$-ranks of the $\mathscr{A}_{K, S}$ are obvious, since $K$ is totally real, giving finite groups except for $S=P$ where $\operatorname{rk}_{\mathbb{Z}_{p}}\left(\mathscr{A}_{K, P}\right)=1$ :

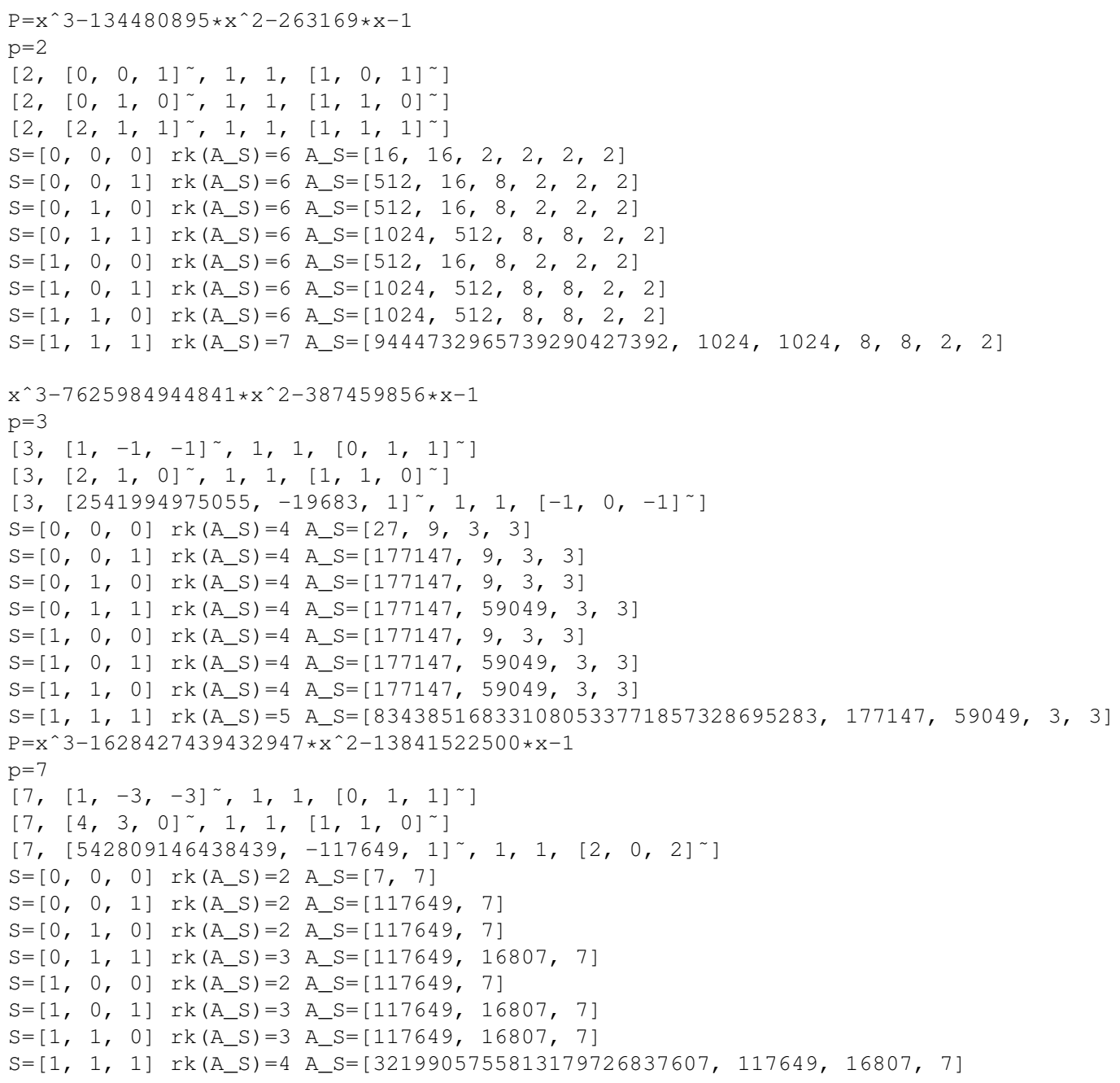

\subsubsection{Example with $p$ totally split in degree 5}

For $P=x^{5}-5, n_{0}=8$, and $p=31$ (totally split) one finds one case of non $S$-rationality:

$$
\mathrm{S}=[1,0,0,0,1] \operatorname{rk}\left(\mathrm{A}_{\mathrm{S}}\right)=1 \mathrm{~A}_{\mathrm{S}}=[961] \text {, i.e., } \widetilde{r}_{K, S}=0, \mathscr{T}_{K, S} \simeq \mathbb{Z} / 31^{2} \mathbb{Z} \text { : }
$$

$[31,[-14,1,0,0,0] \sim, 1,1,[7,-15,10,14,1] \sim]$

$[31,[-7,1,0,0,0] \sim, 1,1,[14,2,-13,7,1] \sim]$

$[31,[3,1,0,0,0] \sim, 1,1,[-12,4,9,-3,1] \sim]$

$[31,[6,1,0,0,0] \sim, 1,1,[-6,1,5,-6,1] \sim]$

$[31,[12,1,0,0,0] \sim, 1,1,[-3,8,-11,-12,1] \sim]$ 


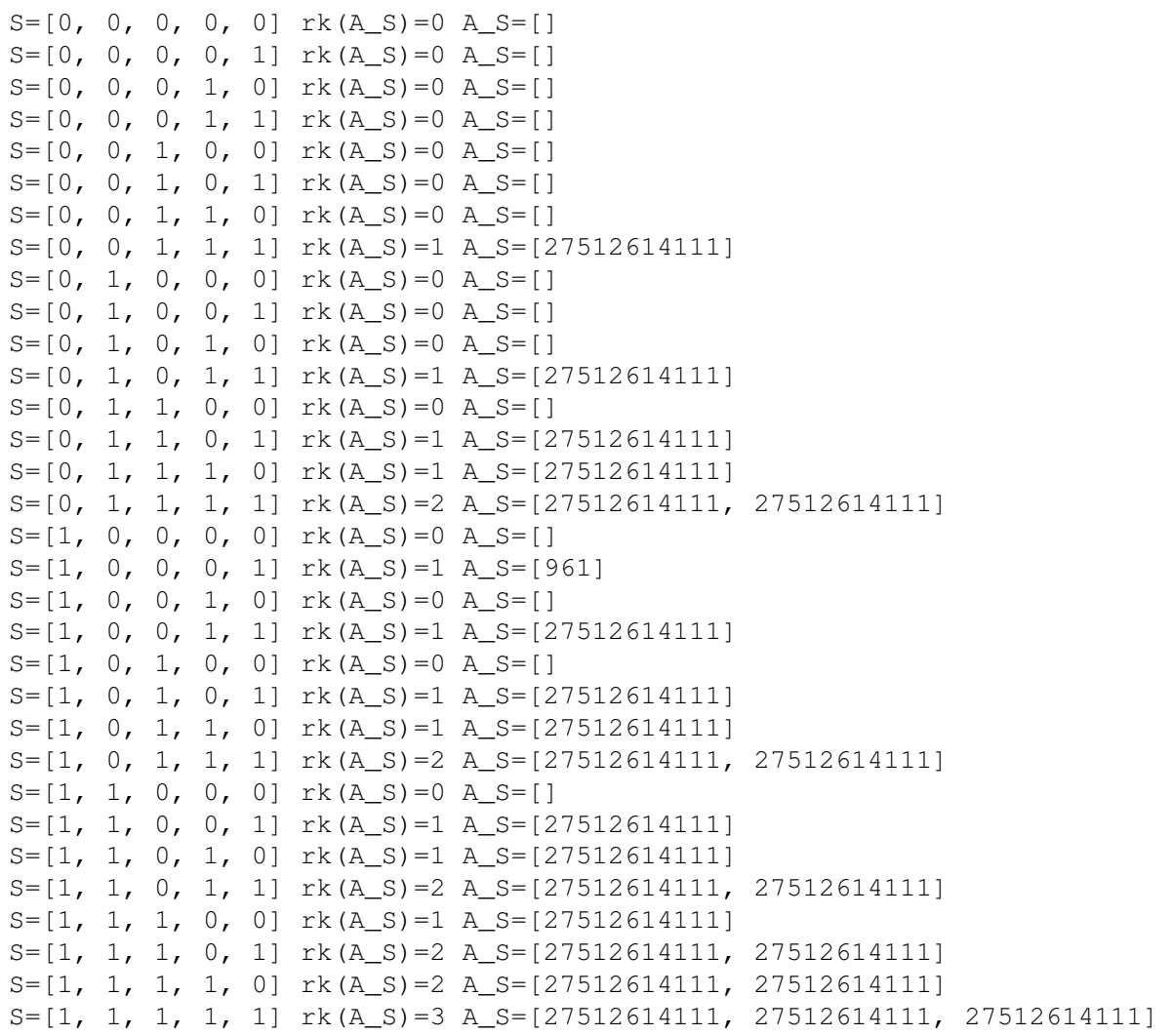

\subsubsection{Example with $p$ totally split in degree 7}

For the polynomial $P=x^{7}-7$ and $p=43$, one finds two cases:

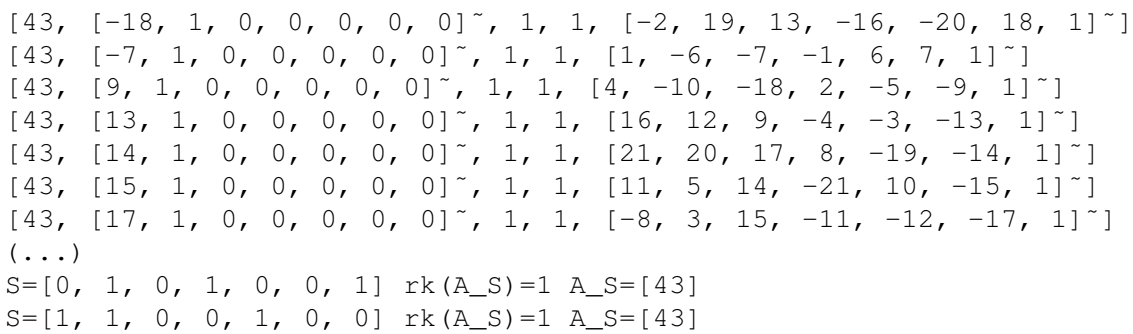

i.e., $\widetilde{r}_{K, S}=0$ and $\mathscr{T}_{K, S} \simeq \mathbb{Z} / 43 \mathbb{Z}$ for the two above cases. For the other modulus, $\mathscr{T}_{K, S}=1$.

\subsubsection{Example with a field discovered by Jaulent-Sauzet}

In [27], some numerical examples of $\{\mathfrak{l}\}(=\{\mathfrak{p}\})$-rational fields, which are not $p$-rational, are given; of course this corresponds to a suitable choice of $S=\{\mathfrak{p}\}$ and we give the case of the field defined by the polynomial:

$$
P=x^{10}+19 x^{8}+8 x^{7}+130 x^{6}+16 x^{5}+166 x^{4}-888 x^{3}-15 x^{2}+432 x+243
$$

for $p=3$ :

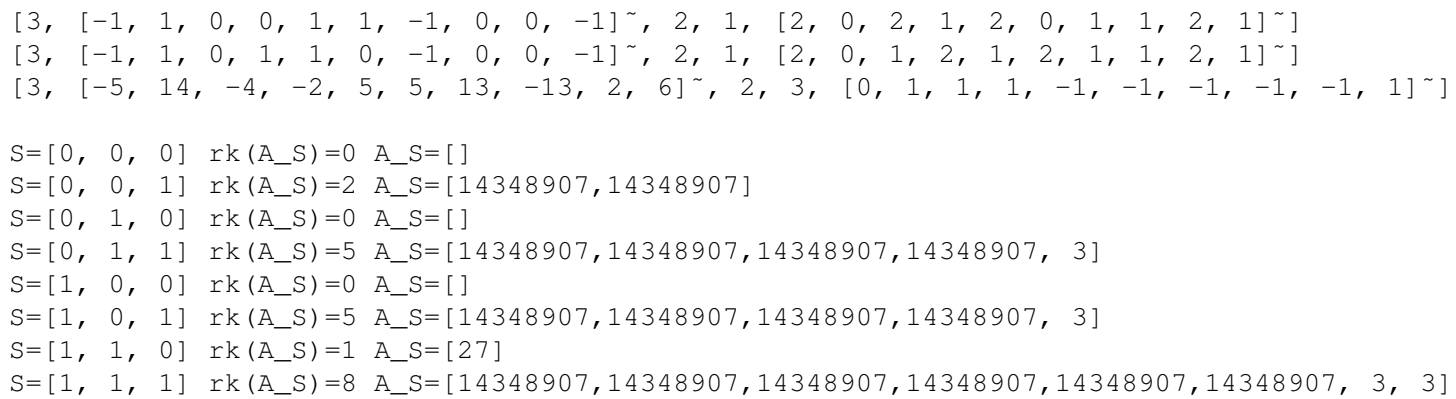


which is indeed $\{\mathfrak{p}\}$-rational for each prime ideal $\mathfrak{p}$, but the field is not 3 -rational since $\mathscr{T}_{K, P} \simeq \mathbb{Z} / 3 \mathbb{Z} \times \mathbb{Z} / 3 \mathbb{Z}$.

Note the case $\mathscr{A}_{K, S}=\mathscr{T}_{K, S} \simeq \mathbb{Z} / 27 \mathbb{Z}$.

Many other numerical examples are available in $[27, \S 3 . c]$.

3.1.6 Abelian fields with $\mathscr{T}_{K, S}=1$ but $\mathscr{T}_{K, P} \neq 1$

We consider for this the cyclotomic field $\mathbb{Q}\left(\mu_{24}\right)$. The following program may be used for any abelian field given by polcyclo $(\mathrm{N})$ or polsubcyclo $(\mathrm{N}, \mathrm{d})$ giving the suitable polynomials of degree $d$ dividing $\varphi(N)$ :

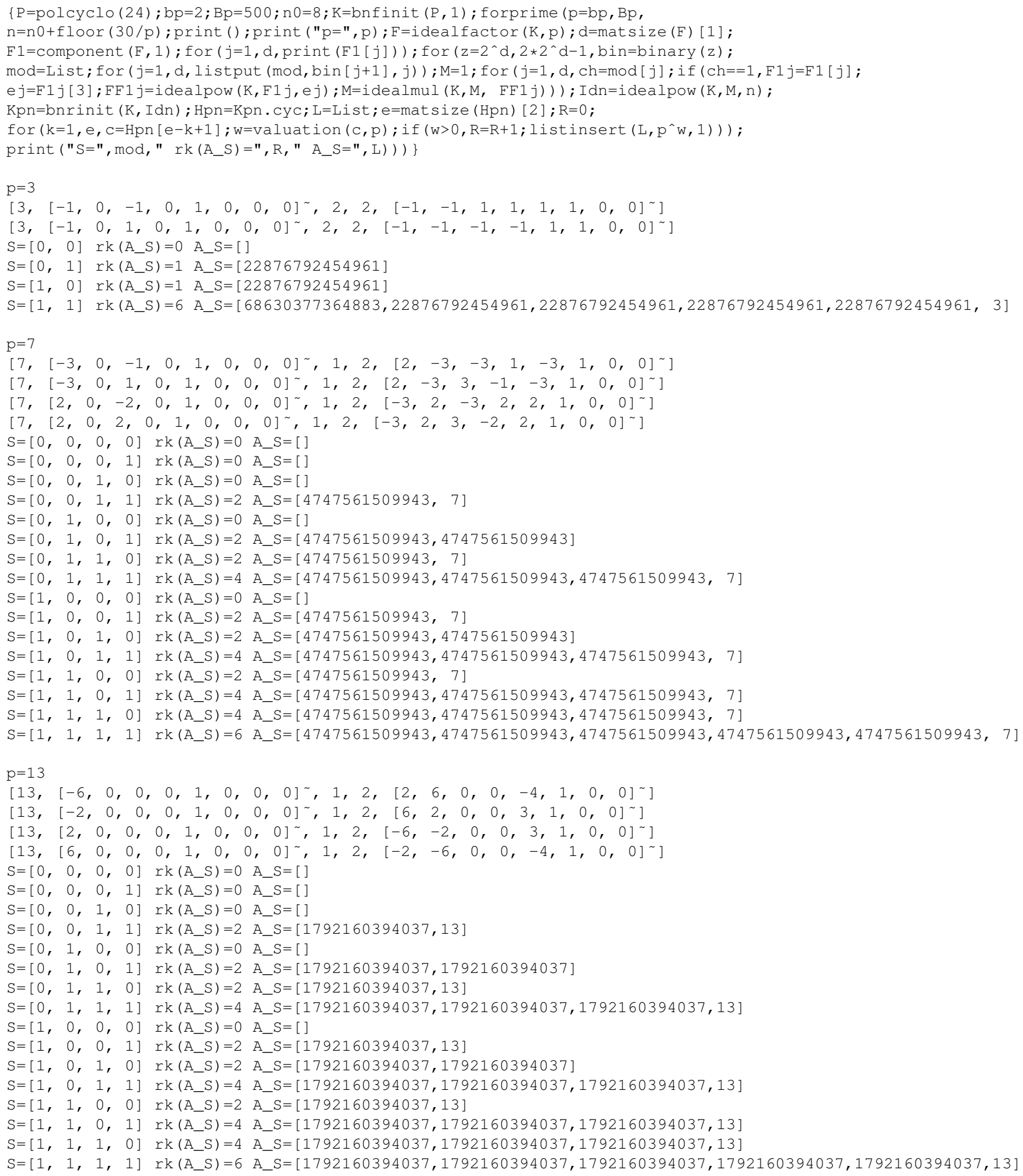




\subsection{Experiments with the fields $K=\mathbb{Q}(\sqrt[p]{N}), N$ prime}

These fields are studied in great detail by Lecouturier in $[31, \S 5]$ for their $p$-class groups and these fields have some remarkable properties. For instance if $\log$ is the discrete logarithm for $(\mathbb{Z} / p \mathbb{Z})^{\times}$provided with a primitive root $g$, the expression $T=\sum_{k=1}^{(N-1) / 2} k \cdot \log (k)(\bmod p)$ governs, under some conditions, the $p$-rank of $C \ell_{K}$ (from a result of Calegari-Emerton, after other similar results of limura, proved again in [31, Theorem 1.1]) and improved by Schaefer-Stubley[32].

So we shall give the general calculations, for all $S \subseteq P$, with that of $T$. We assume $N$ prime congruent to 1 modulo $p$, but the reader may suppress this condition. It seems that many interesting heuristics can be elaborated from the numerical results; we only give some examples (recall that the structure of the class group is given by the first data $S=\emptyset$ ):

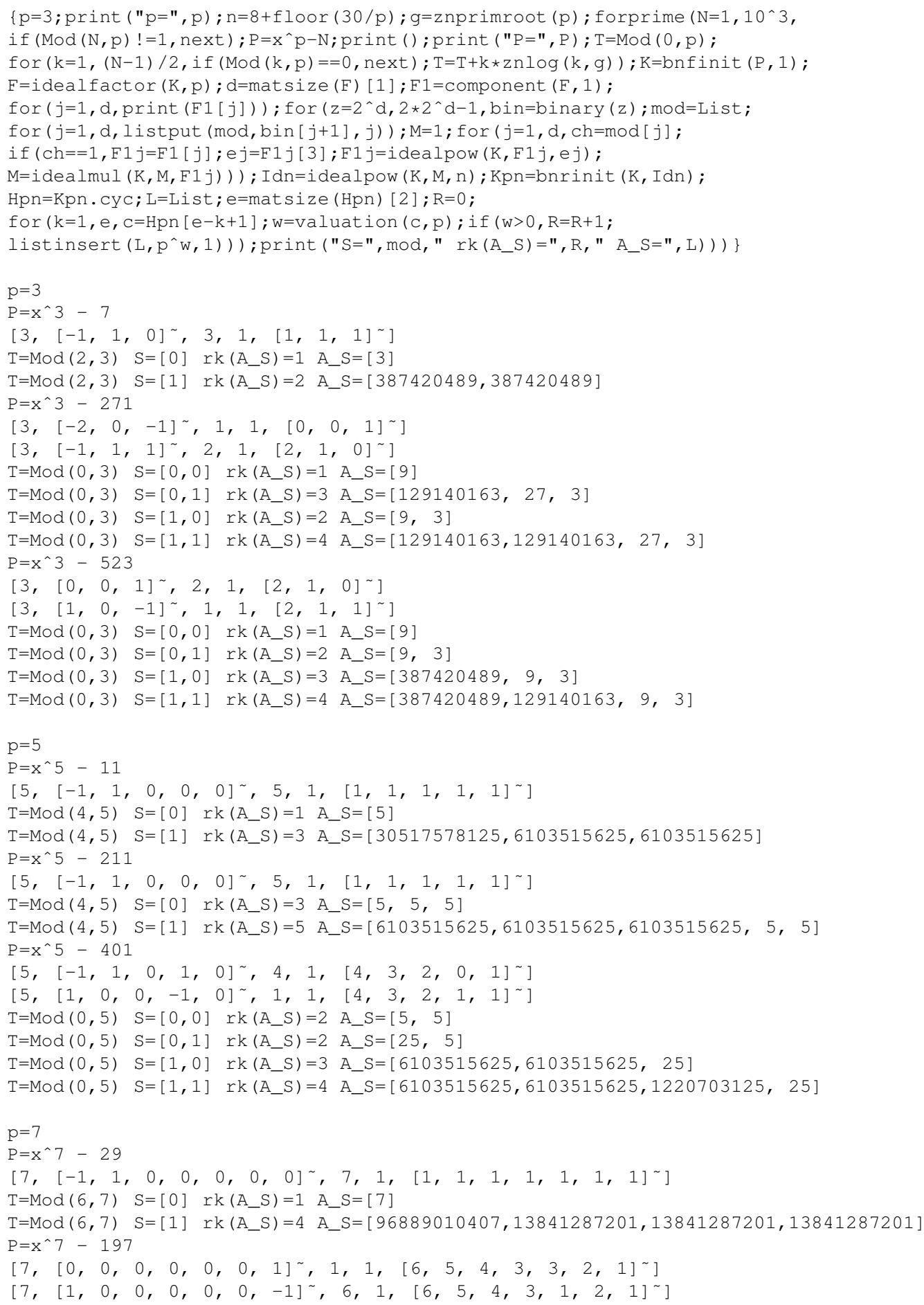




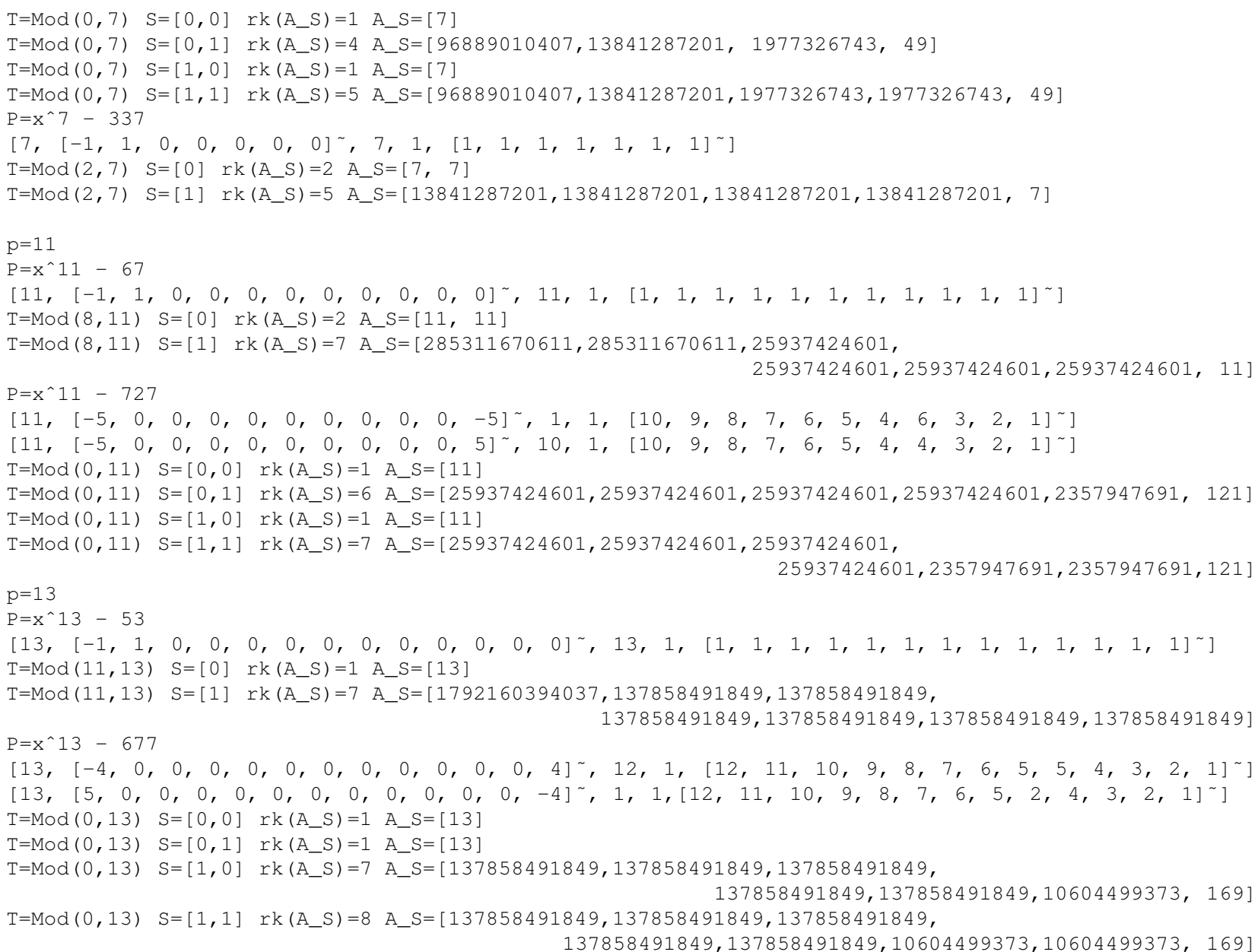

\subsection{The fields $K=\mathbb{Q}(\sqrt{-\sqrt{-q}})$ associated to elliptic curves}

These fields, used by Coates- $\mathrm{Li}$ in $[33,34]$ to prove non-vanishing theorems for the central values at $s=1$ of the complex $L$-series of a family of elliptic curves studied by Gross (for any prime $q \equiv 7(\bmod 8)$ and $p=2)$, are particularly interesting.

Note once for all that the signature of $K$ is $[0,2]$, the Galois closure of $K$ is of degree 8 with Galois group [8, $-1,1$, , $D(4)$ "] and $D_{K}=2^{m} q^{3}$.

\subsubsection{Program for various $p$}

In this part, we fix the prime number $q$ and compute the structure of $\mathscr{A}_{K, S}$ for all sets $S \subseteq P$. Recall that the parameter $n$ must be such that $p^{n}$ be much larger than the exponent of $\mathscr{T}_{K}$.

For instance, for $P=x^{4}+23$, we give the results for $p=3$ and $p=71$ :

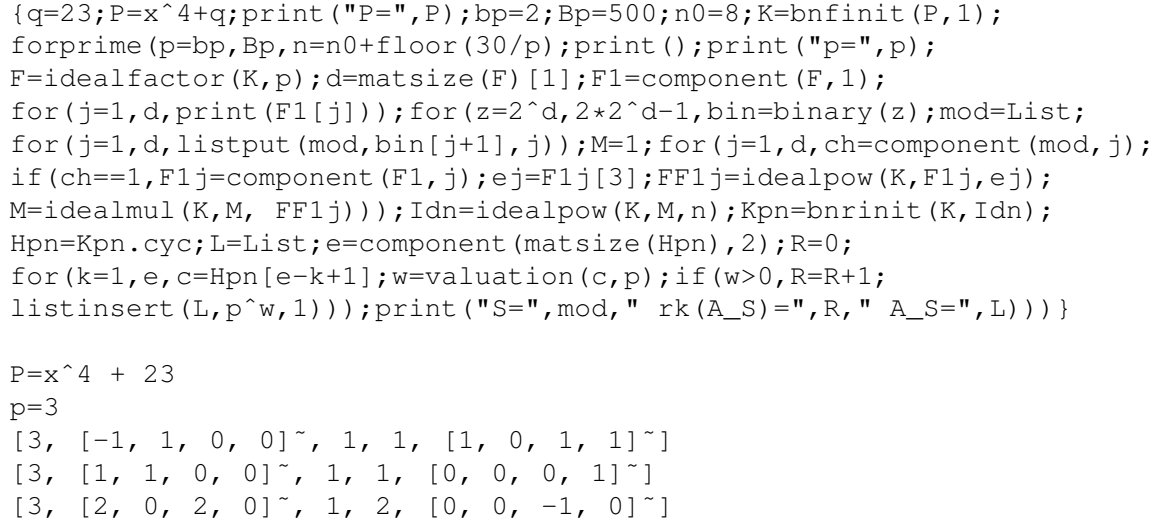




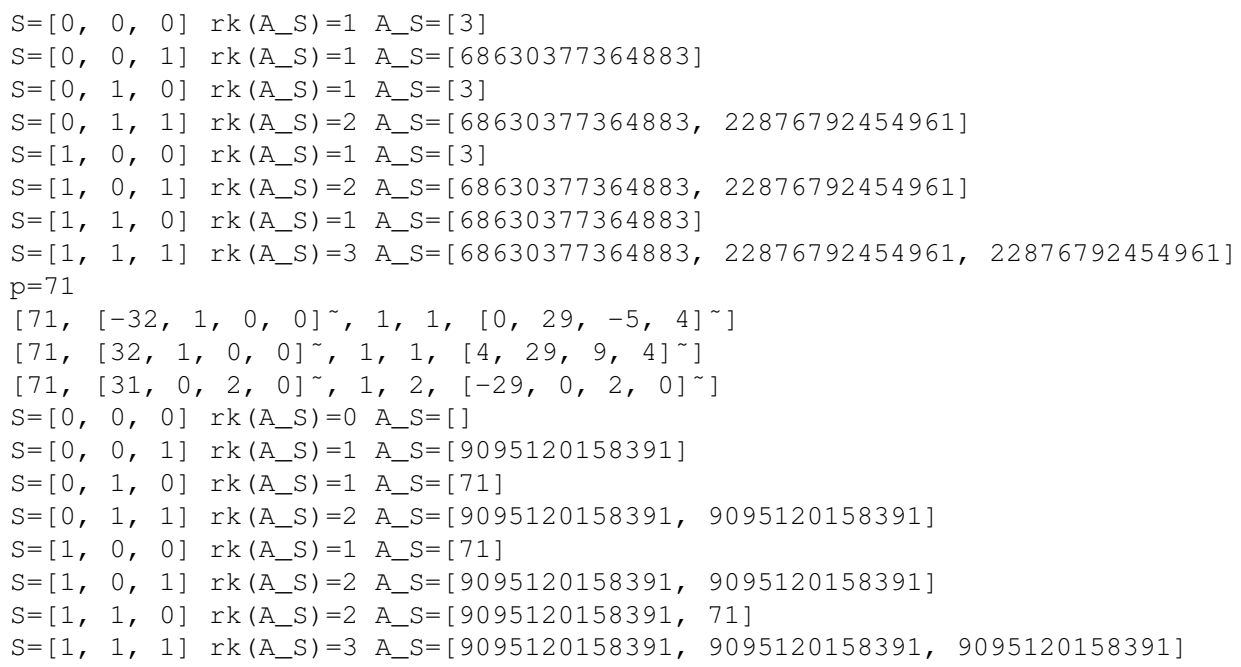

The user is invited to vary $n$ at will to certify the numerical results when the $p$-rank of $\mathscr{A}_{K, S}$ is unknown (i.e., when $S \subsetneq P$ ). In the above examples, some $\mathscr{T}_{K, S}$ are of order $p$ and the $\mathbb{Z}_{p}$-rank of $\mathscr{A}_{K, S}$ is 0 or 1 .

\subsubsection{Program for various $q$ and $p=2$}

The analogous program is the following ( $n=32$ is large enough):

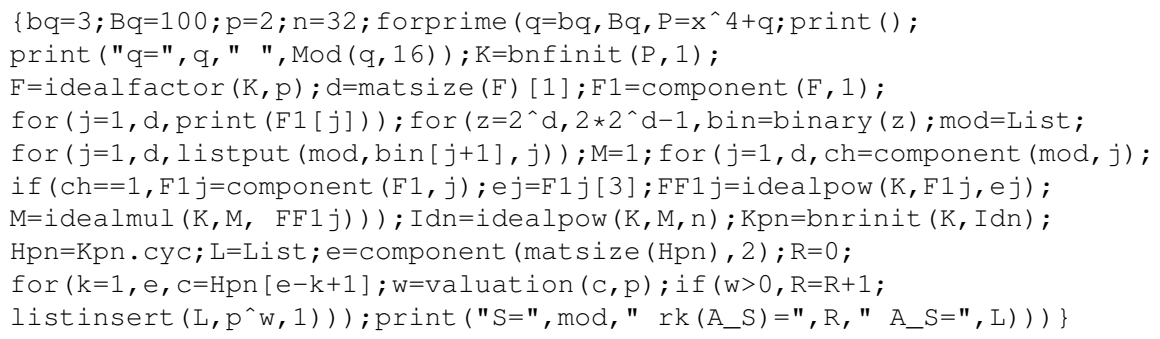

We give an example of each congruence class $q(\bmod 16)$; for $q \equiv 7(\bmod 16)$, the decomposition of $(2)$ in $\mathbb{Q}(\sqrt{-q})$ is $(2)=\mathfrak{p} \cdot \mathfrak{p}^{*}$ where $e_{\mathfrak{p}}=2$ in $K / \mathbb{Q}$ :

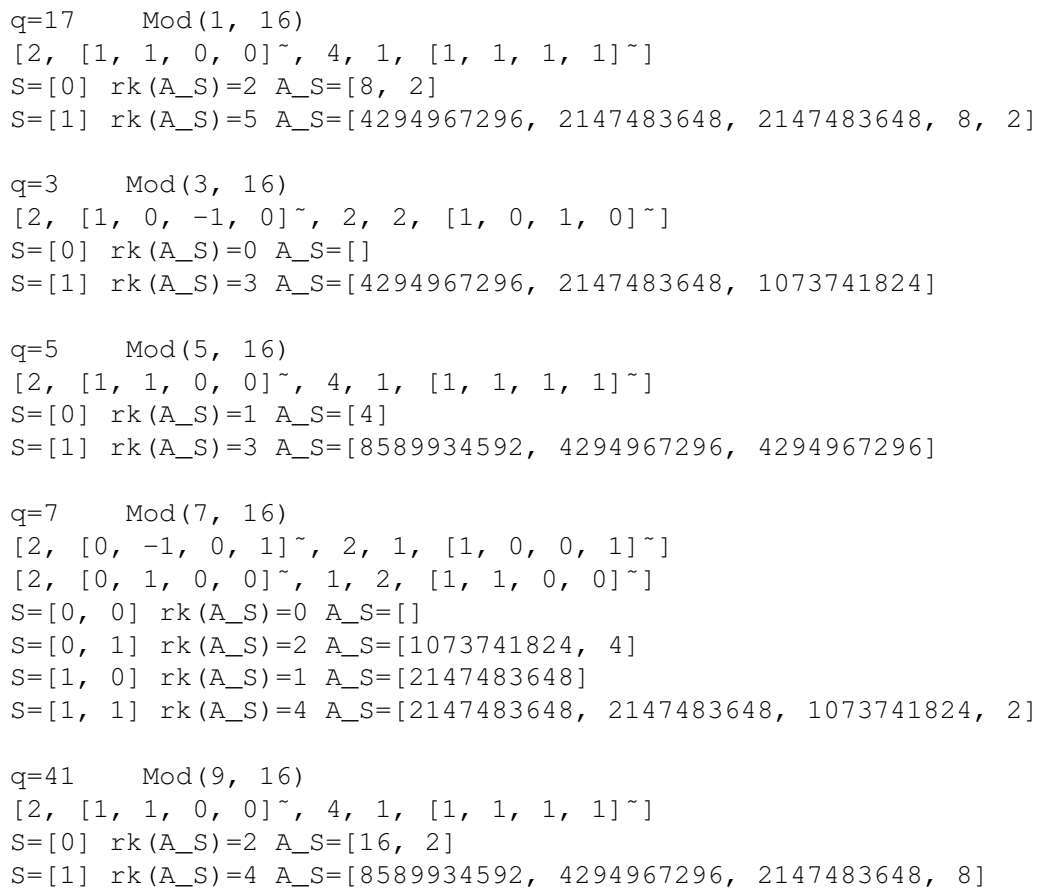




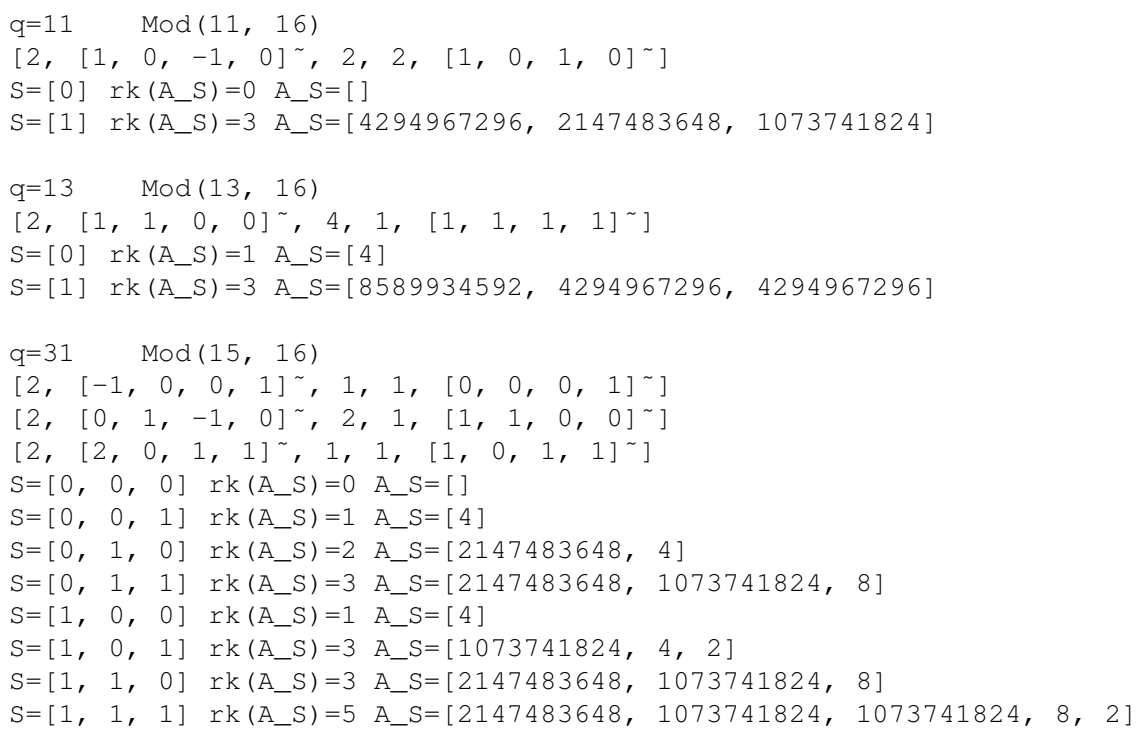

Remark 3.2. A more complete table shows some rules:

(i) For $q \equiv 3(\bmod 8), \mathscr{T}_{K, S}=1$ for $S=\emptyset$ and $S=P=\{\mathfrak{p}\}$;

(ii) For $q \equiv 5(\bmod 8), \mathscr{T}_{K, \emptyset}=C \ell_{K} \simeq \mathbb{Z} / 4 \mathbb{Z}$ and $\mathscr{T}_{K, P}=1$ for $P=\{\mathfrak{p}\}$ (which means that the 2-Hilbert class field of $K$ is contained in the compositum of the $\mathbb{Z}_{2}$-extensions of $\left.K\right)$;

(iii) For $q \equiv 7(\bmod 16)$, for $S=\{\mathfrak{p}\}$ with $e_{\mathfrak{p}}=2$, we get $\mathscr{T}_{K, S} \simeq \mathbb{Z} / 4 \mathbb{Z}$ and for $S=\left\{\mathfrak{p}^{*}\right\}$ with $e_{\mathfrak{p}^{*}}=1$, we get $\mathscr{T}_{K, S}=1$; then $\mathscr{T}_{K, P} \simeq \mathbb{Z} / 2 \mathbb{Z}$.

These properties may be proved easily and are left to the reader as exercises on the $\log _{S}$-function (Definition A.4): consider first the arithmetic of the subfield $k=\mathbb{Q}(\sqrt{-q})$ and use fixed point formulas (A.5) in $K / k$.

(iv) For $q \equiv 15$ ( $\bmod 16)$, the results do not follow any obvious rule and offers some interesting examples as the following ones:

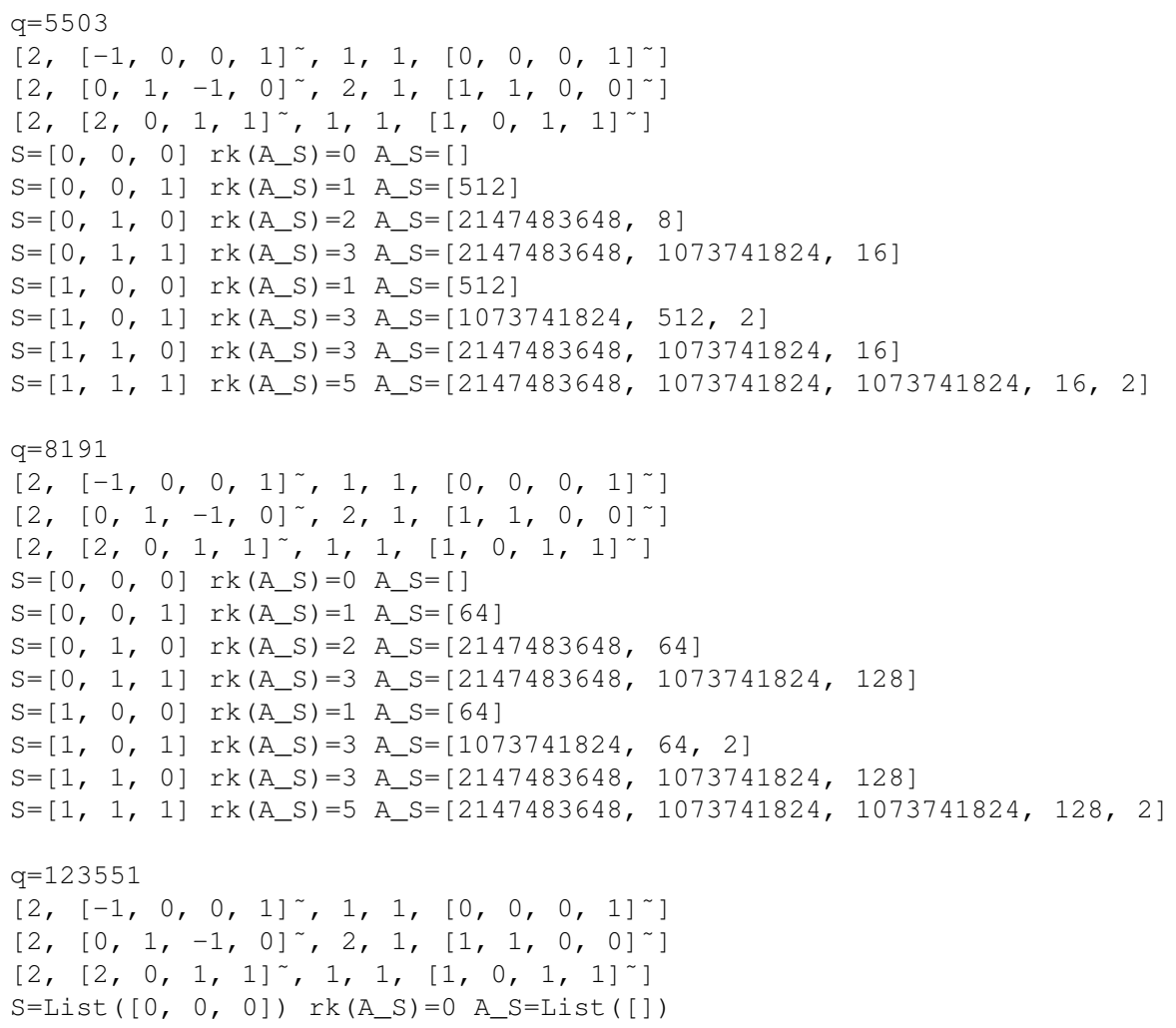




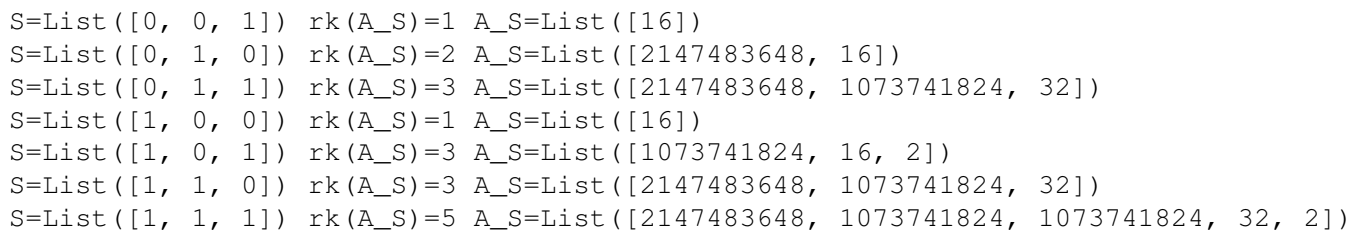

\section{Appendix: History of abelian $p$-ramification}

\section{A.1 Motivations}

We intend, in this detailed survey, to give a maximum of practical information and results about the torsion groups $\mathscr{T}_{K, S}$ that we have numerically computed in the first part of the paper with a PARI/GP program. Since all the invariants, associated with $\mathscr{T}_{K, S}$, need numerical computations for a better understanding, we choose the more suitable technical presentation (the main philosophical remark is that they are all equivalent).

For convenience, we indicate both the original historical contributions and the corresponding results processed systematically in our book [1].

We will not detail the immense domains of pro- $p$-groups and Galois cohomology, whose main purpose is for instance the existence of infinite towers of $S$-ramified extensions and the Fontaine-Mazur conjecture studied by various schools of mathematicians (for this, see, e.g., [5, $\S 10]$ ), nor the analytic aspects as the non-vanishing at $s=1$ of complex $L$-series associated to elliptic curves... Similarly, we shall not consider the context of Iwasawa's theory because this efficient tool does not exempt from having the "basic" arithmetical properties of the corresponding objects.

Note that the solutions of the analogous problems of $S$-ramification over local fields are not sufficient for a "globalization" over a number field $K$ as remarked by Nguyen Quang Do in [35, $\S 9$ ]. Indeed, the global theory depends on Leopoldt's conjecture (usually assumed) and the torsion groups $\mathscr{T}_{K, S}$ are, in some sense, refinements of this conjecture.

So we will focus, mainly, on class field theory and on these specific deep $p$-adic properties or conjectures which are, in our opinion, the main obstructions for many contemporary researches.

We will not give the most general statements but restrict ourselves to the case of $S$-ramification, $S \subseteq P$, without decomposition of finite or infinite places (indeed, in these more elaborate cases, the formalism is identical and may be found in our book). Since the properties of $S$-ramification may be used by many researchers working on different subjects, we will try to explain the numerous steps of its progress. This must be understood for practical information and will be an opportunity to clarify the vocabulary and the main contributions.

We apologize for the probable lack of references (and citation of their authors).

\section{A.2 Prehistory}

The origin of interest for $S$-ramification theory over a number field is probably a paper of Brumer [4], following Serre's book [2] and seems also due to a lecture by Šafarevič (1963) showing the importance of the subject. In [3], Šafarevič gives the cohomological characteristics of the group $\mathscr{G}_{K, S}$ (number of generators and relations, cohomological dimension ...).

Recall at this step the Golod-Šafarevič theorem (1964), named soon after the theorem of Golod-Šafarevič-GaschützVinberg, saying that if a pro-p-group $\mathscr{G}$ is finite, then $r(\mathscr{G})>\frac{1}{4}(d(\mathscr{G}))^{2}$ where $d(\mathscr{G})$ (resp. $r(\mathscr{G})$ ) is the minimal number of generators (resp. relations) for the presentation of $\mathscr{G}$. All of this was developed in Koch's book [36] from the works of many German mathematicians and is amply improved in [5] (see also in Hajir-Maire [37, 38] a good introduction on the subject and some of its developments [39], [40, 41], [42, 43]).

More precisely, in [3, Théorème I], Šafarevič gives, for any number field $K$ and any set of places $S$, the main formula (1.2) that we recall:

\section{A.2.1 Šafarevič formula}

The $p$-rank of the $\mathbb{Z}_{p}$-module $\mathscr{A}_{K, S}$ (giving the minimal number of generators $\operatorname{dim}_{\mathbb{F}_{p}}\left(\mathrm{H}^{1}\left(\mathscr{G}_{K, S}, \mathbb{Z} / p \mathbb{Z}\right)\right.$ ) of $\left.\mathscr{G}_{K, S}\right)$ is:

$$
\operatorname{rk}_{p}\left(\mathscr{A}_{K, S}\right)=\operatorname{rk}_{p}\left(V_{K, S} / K_{(S)}^{\times p}\right)+\sum_{\mathfrak{p} \in S \cap P}\left[K_{\mathfrak{p}}: \mathbb{Q}_{p}\right]+\sum_{\mathfrak{p} \in S} \delta_{\mathfrak{p}}-\delta_{K}-\left(r_{1}+r_{2}-1\right),
$$

where:

$$
K_{(S)}^{\times}:=\left\{\alpha \in K^{\times}, \alpha \text { prime to } S\right\}, \quad V_{K, S}:=\left\{\alpha \in K_{(S)}^{\times},(\alpha)=\mathfrak{a}^{p}\right\},
$$


then $\delta_{\mathfrak{p}}=1$ or 0 according as the completion $K_{\mathfrak{p}}$ contains $\mu_{p}$ or not, and $\delta_{K}=1$ or 0 according as $K$ contains $\mu_{p}$ or not.

Of course, $\operatorname{dim}_{\mathbb{F}_{p}}\left(\mathrm{H}^{2}\left(\mathscr{G}_{K, S}, \mathbb{Z} / p \mathbb{Z}\right)\right)$, giving the minimal number of relations, is easily obtained only when $P \subseteq S$ (equal to $\operatorname{rk}_{p}\left(\mathscr{T}_{K, S}\right)$ under Leopoldt's conjecture), which shall explain the forthcoming studies about this:

[5], [6, 7], [8], [11], [12], [14], [36], [44], Haberland [45], [46], El Habibi-Ziane [47] ....

\section{A.2.2 Kubota formalism}

Mention that Kubota [48] begins the study of the structure of the dual $\mathscr{A}_{K, S}^{*}$ of $\mathscr{A}_{K, S}$, study which is based on the GrunwaldWang theorem and which leads to a characterization of this group in terms of its fundamental invariants called, following Kaplansky, the "Ulm invariants".

Then in [49], Miki uses this formalism, about $\ell(=p)$-ramification, then class field theory, Iwasawa's theory, in direction of Leopoldt's conjecture. Some statements, equivalent to some results that we shall recall in this survey (as well as the notion of $p$-rationality and its main properties), should be mentioned in his paper, despite the difficulty of translating vocabulary and technique.

\section{A.3 Main developments after the pioneering works}

The computation of $\operatorname{rk}_{p}\left(\mathscr{T}_{K, P}\right)$, from Kummer theory, is already given by Bertrandias-Payan [18], then in [50, Théorèmes I.2, I.3, Corollaire 1] and by many authors, for instance by means of cohomological techniques (e.g., [26, Proposition 3]).

This will give reflection formulas.

\section{A.3.1 Reflection and rank formulas}

From [51, Chapitre III, $\S 10]$ or [1, $\S$ II.5.4.1][Gr2003]. Using the Šafarevič formula and Kummer theory when $K$ contains the group $\mu_{p}$ of $p$ th roots of unity, and writing (for $S \subseteq P$ ):

$$
P=S \cup \Sigma \text { with } S \cap \Sigma=\emptyset,
$$

one obtains the reflection theorem in its simplest form:

$$
\operatorname{rk}_{p}\left(\mathscr{A}_{K, S}^{\Sigma}\right)-\operatorname{rk}_{p}\left(\mathscr{A}_{K, \Sigma}^{S \text { res }}\right)=\# S-\# \Sigma+\sum_{\mathfrak{p} \in S}\left[K_{\mathfrak{p}}: \mathbb{Q}_{p}\right]-r_{1}-r_{2}
$$

where $\mathscr{A}_{K, S}^{\Sigma}$ is the Galois group of the maximal abelian pro- $p$-extension of $K$ in $H_{K, S}$, which is $\Sigma \cup\{\infty\}$-split (i.e., in which all the places of $\Sigma \cup\{\infty\}$ split completely), and similarly for the definition of $\mathscr{A}_{K, \Sigma}^{\text {Sres }}$, in the restricted sense for $p=2$ (i.e., only $S$-split); in other words, the mention of $\{\infty\}$ is implicit in the upper script to give the ordinary sense when $p=2$.

The case $S=P$ leads to the following well-known result:

Theorem A.1. [1, Proposition III.4.2.2]. Let $K$ be any number field fulfilling the Leopoldt conjecture for the prime number $p$. Let $K^{\prime}:=K\left(\mu_{p}\right), P^{\prime}$ be the set of $p$-places above $P$ in $K^{\prime}$, and let $P^{\mathrm{dec}}$ be the set of $p$-places of $K$ totaly split in $K^{\prime}$. Let $\omega$ be the Teichmüller character and denote by $\mathrm{rk}_{\omega}$ the p-rank of an isotypic $\omega$-component for $\operatorname{Gal}\left(K^{\prime} / K\right)$; then:

$$
\operatorname{rk}_{p}\left(\mathscr{T}_{K, P}\right)=\operatorname{rk}_{\omega}\left(C \ell_{K^{\prime}}^{P^{\prime} \text { res }}\right)+\# P^{\mathrm{dec}}-\delta_{K}
$$

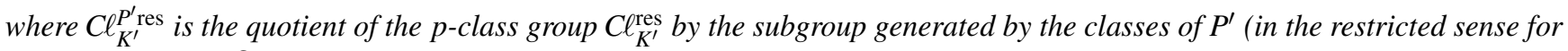
$p=2$ ) and where $\delta_{K}=1$ or 0 according as $K$ contains $\mu_{p}$ or not. Whence the following properties:

(i) If $\mu_{p} \subset K$, we then have $\operatorname{rk}_{p}\left(\mathscr{T}_{K, P}\right)=\operatorname{rk}_{p}\left(C \ell_{K}^{P \text { res }}\right)+\# P-1$.

(ii) We have $\mathscr{T}_{K, P}=1$ if and only if:

- $\mu_{p} \not \subset K($ so $p \neq 2)$ : then $P^{\mathrm{dec}}=\emptyset$ and the $\omega$-component of $C \ell_{K^{\prime}}$ is trivial;

- $\mu_{p} \subset K: p$ does not split in $K / \mathbb{Q}$ and the unique $\mathfrak{p} \in P$ generates $C \ell_{K}^{\mathrm{res}}$.

Example A.2. For $K=\mathbb{Q}\left(\mu_{p}\right)=: \mathbb{Q}\left(\zeta_{p}\right), p \neq 2$, taking $\Sigma=\emptyset$ and $S=P$ :

$$
\mathrm{rk}_{p}\left(\mathscr{A}_{K, P}\right)-\mathrm{rk}_{p}\left(\mathscr{A}_{K, \emptyset}^{P}\right)=1+p-1-\frac{p-1}{2}=\frac{p+1}{2} .
$$

Since $\mathscr{A}_{K, \emptyset}^{P}=C \ell_{K} /\left\langle\ell_{K}(\mathfrak{p})\right\rangle$, with $\mathfrak{p}=\left(1-\zeta_{p}\right)$, and $\mathscr{A}_{K, P} \simeq \mathbb{Z}_{p}^{\frac{p+1}{2}} \bigoplus \mathscr{T}_{K, P}$, this yields:

$$
\operatorname{rk}_{p}\left(\mathscr{T}_{K, P}\right)=\operatorname{rk}_{p}\left(C \ell_{K}\right)
$$

as well as the writing $\operatorname{rk}_{p}\left(\mathscr{T}_{K, P}^{ \pm}\right)=\operatorname{rk}_{p}\left(C \ell_{K}^{\mp}\right)$ (for analogous equalities with pairs of isotopic components associated by means of the mirror involution, and the consequences for Vandiver's conjecture, see [52]). 
If the condition $S \cup \Sigma=P$ is not fulfilled, we have (still assuming $\mu_{p} \subset K$ ) the reflection formula:

$$
\operatorname{rk}_{p}\left(\mathscr{A}_{K, S}^{\Sigma}\right)-\operatorname{rk}_{p}\left(C \ell_{K}^{S \operatorname{Sres}}\left(\mathfrak{m}^{*}\right)\right)=\# S-\# \Sigma+\sum_{\mathfrak{p} \in S}\left[K_{\mathfrak{p}}: \mathbb{Q}_{p}\right]-r_{1}-r_{2}, \text { with } \mathfrak{m}^{*}:=\prod_{\mathfrak{p} \in \Sigma} \mathfrak{p}^{p e_{\mathfrak{p}}+1} \cdot \prod_{\mathfrak{p} \in P \backslash S \cup \Sigma} \mathfrak{p}^{p e_{\mathfrak{p}}}
$$

where $C \ell_{K}^{\text {Sres }}\left(\mathfrak{m}^{*}\right)$ is the $S$-split $p$-ray class group of modulus $\mathfrak{m}^{*}$ (see [1, Exercise II.5.4.1, proof of (iii)] and (iv) for the case $p=2)$. Note that $C \ell_{K}^{\text {Sres }}\left(\mathfrak{m}^{*}\right)$ is isomorphic to a quotient of $\mathscr{A}_{K, P \backslash S}^{\text {Sres }}$.

Finaly, if $K$ does not contain $\mu_{p}$, but assuming $P=S \cup \Sigma$ with $S \cap \Sigma=\emptyset$, the general formula is:

$$
\operatorname{rk}_{p}\left(\mathscr{T}_{K, S}^{\Sigma}\right)=\operatorname{rk}_{\omega}\left(\mathscr{A}_{K^{\prime}, \Sigma^{\prime}}^{S^{\prime} \text { res }}\right)+\sum_{\mathfrak{p} \in S} \delta_{\mathfrak{p}}-\delta_{K}-\# \Sigma-\left(r_{1}+r_{2}-1-r_{K, S}^{\Sigma}\right),
$$

where:

$$
r_{K, S}^{\Sigma}=\sum_{\mathfrak{p} \in S}\left[K_{\mathfrak{p}}: \mathbb{Q}_{p}\right]-\widetilde{r}_{K, S}^{\Sigma}
$$

here, $\widetilde{r}_{K, S}^{\Sigma} \leq r_{2}+1$ is the $\mathbb{Z}_{p}$-rank of $\mathbb{Z}_{p} \log _{S}\left(I_{K, S}\right)$ modulo $\mathbb{Q}_{p} \log _{S}\left(E_{K}^{\Sigma}\right)$ dealing with the group $E_{K}^{\Sigma}$ of $\Sigma$-units of $K$ (see also [11], [14] for some applications).

One can restrict some of the above equalities to $p$-class groups, giving only inequalities on the $p$-ranks (Hecke theorem (1910), Scholz theorem (1932), Leopoldt Spiegelungssatz (1958), Armitage-Fröhlich-Serre, Oriat, for $p=2$.

For reflection theorems and formulas with characters, see [1, II.5.4, Theorem II.5.4.5)] from the computations of [51, Ch. I, Theorem 5.18] where $p$-rank formulas link $p$-class groups and torsion groups as in Theorem A.1 (this context is used by Ellenberg-Venkatesh in [53] for the $\varepsilon$-conjecture on $p$-class groups).

For the annihilation of the Galois module $\mathscr{T}_{K, P}$, of real abelian extensions $K / \mathbb{Q}$, in relation with the construction of $p$-adic $L$-functions and reflection principle, see [54] and its bibliography. There is probably equivalent information whatever the process (algebraic or analytic), as shown by Oriat in [55]. This logical aspect should deserve further investigation.

\section{A.3.2 Regulators and $p$-adic residues of the $\zeta_{p}$-functions}

We continue the story with the $p$-adic analytic computations of the residue of the $p$-adic $\zeta$-function at $s=1$ of real abelian fields $K$ by Amice-Fresnel [56], from Kubota-Leopoldt $L_{p}$-functions (1964), by Coates [57], Serre [58] introducing $p$-adic pseudo-measures, then by Colmez [59] in full generality, via the formula:

$$
\frac{1}{2^{[K: \mathbb{Q}]-1}} \lim _{s \rightarrow 1}(s-1) \zeta_{K, p}(s)=\frac{R_{p} h E_{p}(1)}{\sqrt{D}},
$$

where $R_{p}$ is the classical $p$-adic regulator, $h$ the class number, $D$ the discriminant of $K$ and $E_{p}(1)$ the eulerian factor $\prod_{\mathfrak{p} \mid p}(1-$ $\mathrm{Np}^{-1}$ ). For totally real fields, the normalised $p$-adic regulator $\mathscr{R}_{K, P}$, in the formula (2.2), is given (under Leopoldt's conjecture) by the expression [19, Proposition 5.2]:

$$
\# \mathscr{R}_{K, P} \sim \frac{1}{2} \cdot \frac{\left(\mathbb{Z}_{p}: \log \left(\mathrm{N}_{K / \mathbb{Q}}\left(U_{K, P}\right)\right)\right)}{\# \mathscr{W}_{K, P} \cdot \prod_{\mathfrak{p} \mid p} \mathrm{~Np}} \cdot \frac{R_{p}}{\sqrt{D}},
$$

where $\sim$ means equality up to a $p$-adic unit factor; whence:

$$
\frac{1}{2^{[K: \mathbb{Q}]-1}} \lim _{s \rightarrow 1}(s-1) \zeta_{K, p}(s)=\frac{1}{p\left[K \cap \mathbb{Q}^{\mathrm{c}}: \mathbb{Q}\right]} \# \mathscr{T}_{K, P},
$$

where $\mathbb{Q}^{c}$ is the $\mathbb{Z}_{p}$-cyclotomic extension of $K$. In [120], Hatada uses the link between the $p$-adic valuation of $\zeta_{K}(2-p)$ and that of $\mathscr{R}_{K, P}$ to study the $p$-rationality of some totally real number fields; he studies the case of quadratic fields with general Fibonacci sequences (from the fundamental unit), a method that will be rediscovered by some authors to characterize the $p$-rationality.

Mention the relative version of the Coates formula in the totally real case:

Theorem A.3. [50, Théorème III.3]. Let L/K be an abelian extension of totally real number fields fulfilling the Leopoldt conjecture. Let $\mathscr{N}_{L / K}$ be the group of local norms and let $C \ell_{L / K}^{\mathrm{gen}}:=\operatorname{Gal}\left(H_{L}^{\mathrm{ab}} / L H_{K}\right)$ be the p-genus group in $L / K$; the superscript ${ }^{*}$ denotes $\operatorname{Ker}\left(\mathrm{N}_{L / K}\right)$. Then:

$$
\begin{aligned}
\# \mathscr{T}_{L, P} \sim \frac{\# \mathscr{T}_{K, P}}{\left[L \cap H_{K, P}: L \cap K^{\mathrm{c}}\right]} \times \frac{\prod_{\mathrm{r} \nmid p} e_{\mathfrak{l}, p}}{\left[L: L \cap H_{K, P}\right]} & \times{ }^{\mathrm{gen}} \times \ell_{L / K}^{\mathrm{gen}} \times\left(E_{K} \cap \mathscr{N}_{L / K}: \mathrm{N}_{L / K}\left(E_{L}\right)\right) \\
& \times\left(\log _{P}\left(U_{L, P}^{*}\right): \log _{P}\left(\bar{E}_{L}^{*}\right)\right) \times\left(\operatorname{tor}_{\mathbb{Z}_{p}}\left(U_{L, P}^{*}\right): \mu_{p}^{*}\right),
\end{aligned}
$$

where $\mu_{p}^{*}=1$ for $p \neq 2$ and $\# \mu_{2}^{*}=\operatorname{gcd}(2,[L: K])$. 


\section{A.3.3 Cohomological interpretation}

In [16], Nguyen Quang Do gives the cohomological interpretation of the dual of $\mathscr{T}_{K, P}: \mathscr{T}_{K, P}^{*} \simeq \mathrm{H}^{2}\left(\mathscr{G}_{K, P}, \mathbb{Z}_{p}\right)$, considered as the first of the mysterious non positive twists $\mathrm{H}^{2}\left(\mathscr{G}_{K, P}, \mathbb{Z}_{p}(i)\right)$ of the motivic cohomology; for concrete results of genus type about the corresponding case of motivic tame kernels, see Assim-Movahhedi [60] and its important bibliography which would deserve to be in part among our references, despite it is beyond our goals.

It is indeed well known that $\mathrm{H}^{2}\left(\mathscr{G}_{K, P}, \mathbb{Z}_{p}\right)$ does appear as a tricky obstruction in many questions of Galois theory over number fields, whatever the technical approach. For $\mathrm{H}^{2}\left(\mathscr{G}_{K, S}, \mathbb{Z}_{p}\right)$, see $[1$, Appendix][Gr2003].

But considering the two "equivalent" invariants $\mathrm{H}^{2}\left(\mathscr{G}_{K, P}, \mathbb{Z}_{p}\right)$ and $\mathscr{T}_{K, P}$, only the last one may be used, with arithmetic or analytic tools, to obtain numerical experiments and to understand the true intrinsic $p$-adic difficulties.

\section{A.3.4 Principal Conjectures and Theorems}

Considering the invariants $C \ell_{K}$ and $\mathscr{T}_{K, P}$ as fundamental objects, we have given, for the abelian fields $K$, the conjectural behavior of their isotopic $\chi$-components for irreducible $p$-adic characters $\chi$ in [61]; the proofs of these conjectures and of some improvements in Iwasawa's theory are well known and the reader may refer to the illuminating paper of Ribet [62] (available at https: / /www.dropbox.com/s/1uir9crhidorejy/smf.Ribet.pdf?dl=0) about the so-called "Principal Theorem" stemming from Bernoulli-Kummer-Herbrand then Ribet-Mazur-Wiles-Thaine-Rubin-Kolyvagin-Greither works on cyclotomy and $p$-adic $L$-functions, as a prelude of wide generalizations in the same spirit.

\section{A.4 Basic $p$-adic properties of $\mathscr{A}_{K, P} \& \mathscr{T}_{K, P}$}

During the 1980 's, we have written in $[50,63,64]^{3}$ the main properties of the groups $\mathscr{T}_{K, P}$ with their behaviour in any extension $L / K$ and proved (assuming Leopoldt's conjecture in the Galois closure of $L$ ) that the transfer maps:

$$
\mathscr{A}_{K, P} \longrightarrow \mathscr{A}_{L, P} \& \mathscr{T}_{K, P} \longrightarrow \mathscr{T}_{L, P}
$$

are always injective [50, Théorème I.1]; which has major consequences for the arithmetic of number fields (e.g., non-capitulation in an extension contrary to class groups). Of course, this property has been obtained soon after by Jaulent, Nguyen Quang Do and others with different techniques.

\section{A.4.1 The $p$-adic $\log _{S}$-functions}

Definition A.4. [63, $\S 2$, Théorème 2.1], [1, § III.2.2]. Let $I_{K, P}$ be the group of prime to $p$ ideals of K. We define the logarithm function:

$$
\log _{P}: I_{K, P} \longrightarrow\left(\bigoplus_{\mathfrak{p} \in P} K_{\mathfrak{p}}\right) / \mathbb{Q}_{p} \log _{P}\left(E_{K}\right)
$$

as follows. For any ideal $\mathfrak{a} \in I_{K, P}$ let $m$ be such that $\mathfrak{a}^{m}=:(\alpha), \alpha \in K^{\times}$, then:

$$
\log _{P}(\mathfrak{a}):=\frac{1}{m} \log _{P}(\alpha) \quad\left(\bmod \mathbb{Q}_{p} \log _{P}\left(E_{K}\right)\right)
$$

The main property of $\log _{P}$ is that for any ideal $\mathfrak{a} \in I_{K, P}, \log _{P}(\mathfrak{a})$ defines the Artin symbol in the compositum $\widetilde{K}^{P}$ of the $\mathbb{Z}_{p}$-extensions of $K$ by means of the canonical exact sequence:

$$
1 \rightarrow \mathscr{T}_{K, P} \longrightarrow \mathscr{A}_{K, P} \stackrel{\log _{P}}{\longrightarrow} \log _{P}\left(I_{K, P}\right) \simeq \operatorname{Gal}\left(\widetilde{K}^{P} / K\right) \rightarrow 1
$$

which may be generalized with arbitrary $S \subseteq P$ :

$$
1 \rightarrow \mathscr{T}_{K, S} \longrightarrow \mathscr{A}_{K, S} \stackrel{\log _{S}}{\longrightarrow} \log _{S}\left(I_{K, S}\right) \simeq \operatorname{Gal}\left(\widetilde{K}^{S} / K\right) \rightarrow 1,
$$

with an obvious definition of $\log _{S}(\mathfrak{a})$ in $\bigoplus_{\mathfrak{p} \in S} K_{\mathfrak{p}}$ modulo $\mathbb{Q}_{p} \log _{S}\left(E_{K}\right)$.

This formalism is equivalent to that given by the theory of pro- $p$-groups (here $\mathscr{G}_{K, P}$ ), but may yield numerical computations as follows:

${ }^{3}[64]$ is only available at: https://www.dropbox.com/s/fusia63znk0kcky/Lectures1982.pdf?dl=0 
The formula for $\# \mathscr{T}_{K, S}, S \subseteq P$, is the following [65, Theorems III.2.5], [1, Corollary III.2.6.1] (under Leopoldt's conjecture):

$$
\# \mathscr{T}_{K, S}=\# \mathscr{W}_{K, S} \times \# \mathscr{R}_{K, S} \times \frac{\# C \ell_{K}}{\left(\mathbb{Z}_{p} \log _{S}\left(I_{K, S}\right): \mathbb{Z}_{p} \log _{S}\left(P_{K, S}\right)\right)},
$$

where $P_{K, S}$ is the group of principal ideals prime to $S$, so that $\mathbb{Z}_{p} \log _{S}\left(P_{K, S}\right)$ depends obviously on $\log _{S}\left(U_{K, S}\right)$ modulo $\mathbb{Q}_{p} \log _{S}\left(E_{K}\right)$. When $S \subsetneq P, \mathscr{W}_{K, S}$ is not necessarily equal to tor $\mathbb{Z}_{p}\left(U_{K, S}\right) / \imath_{S}\left(\mu_{K}\right)$ (cf. Lemmas $2.1,2.2$ ).

The denominator in (A.6) gives the degree $\left[\widetilde{K}^{S} \cap H_{K}: K\right]$ and the quotient gives $\#{\widetilde{C \ell_{K}}}_{K}$.

For $S=P$, the $\log _{P}$-function allows, when $\mu_{p} \subset K$, the numerical determination of the initial Kummer radical contained in $\widetilde{K}^{P}$ [66], [67].

\section{A.4.2 Fixed point formula}

Then we have obtained a fixed point formula for $S=P$ which, contrary to Chevalley's formula for class groups in cyclic extensions [68], does exist whatever the Galois extension $L / K([63, \S 5]$, [69, Section 2 (c)], [65, Proposition 6], [25, Appendice I], [70, Appendice]):

Theorem A.5. [1, $\S$ IV.3, Theorem 3.3]. Let $L / K$ be a Galois extension of number fields and $G:=\mathrm{Gal}(L / K)$. Let $p$ be a prime number; we assume that $L$ satisfies the Leopoldt conjecture for $p$. Then:

$$
\# \mathscr{T}_{L, P}^{G}=\# \mathscr{T}_{K, P} \times \frac{\prod_{\mathfrak{l} p p} e_{\mathfrak{l}, p}}{\left(\sum_{\mathfrak{l} \nmid p} \frac{1}{e_{\mathfrak{l}, p}} \mathbb{Z}_{p} \log _{P}(\mathfrak{l})+\mathbb{Z}_{p} \log _{P}\left(I_{K, P}\right): \mathbb{Z}_{p} \log _{P}\left(I_{K, P}\right)\right)},
$$

where $e_{\mathfrak{l}, p}$ is the p-part of the ramification index of $\mathfrak{l}$ in $L / K$.

Remark A.6. Contrary to the computation of tor $_{\mathbb{Z}_{p}}\left(U_{K, P} / \bar{E}_{K}^{P}\right)$, that of the $\mathbb{Q}_{p}$-vector space $\mathbb{Q}_{p} \log _{P}\left(E_{K}\right)$ does not need the knowledge of the group of units $E_{K}$; it only depends of Leopoldt's conjecture (assumed) and its $\mathbb{Q}_{p}$-dimension is $r_{1}+r_{2}-1$; the case of $\mathbb{Q}_{p} \log _{S}\left(E_{K}\right)$ is more mysterious.

The case of totally real fields is easier since the Log-function trivializes because we have $\bigoplus_{\mathfrak{p} \in P} K_{\mathfrak{p}}=\mathbb{Q}_{p} \log _{P}\left(E_{K}\right) \oplus \mathbb{Q}_{p}$, which allows explicit computations [50, Théorème III.1]:

Corollary A.7. [1, Exercise IV.3.3.1]. In the case of a totally real number field $L$, the above formula becomes (under Leopoldt's conjecture): \# $\mathscr{T}_{L, P}^{G}=\# \mathscr{T}_{K, P} \cdot p^{\rho-r} \cdot \prod_{\uparrow \nmid p} e_{\mathfrak{l}, p}$, where $p^{r} \sim[L: K]$ and $\rho$ only depends on the decomposition of the ramified primes $\ell \nmid p$ in $L / K$.

\section{A.4.3 $p$-primitive ramification}

The fixed point formula of Theorem A.5 allows to characterize the case where \# $\mathscr{T}_{L, P}=1$ in a $p$-extension $L / K$ :

Corollary A.8. Let $L / K$ be any finite p-extension. Then $\mathscr{T}_{L, P}=1$ if and only if the two following conditions are fulfilled (under Leopoldt's conjecture):

(i) $\mathscr{T}_{K, P}=1$;

(ii) $\left(\sum_{\mathfrak{l} \nmid p} \frac{1}{e_{\mathfrak{l}, p}} \mathbb{Z}_{p} \log _{P}(\mathfrak{l})+\mathbb{Z}_{p} \log _{P}\left(I_{K, P}\right): \mathbb{Z}_{p} \log _{P}\left(I_{K, P}\right)\right)=\prod_{\mathfrak{l} \nmid p} e_{\mathfrak{l}, p}$.

Definition A.9. $[1, \S I V .3,(b)]$. When the condition (ii) is fulfilled, we say that the p-extension $L / K$ is p-primitively ramified and that the set $T$ of tame places $\mathfrak{l}$, ramified in $L / K$, is primitive [65, Ch. III, Definition \& Remark], which is equivalent (in terms of Frobenius automorphisms) to:

$$
\operatorname{Gal}\left(\widetilde{K}^{P} / K\right) \simeq \mathscr{A}_{K, P} / \mathscr{T}_{K, P}=\bigoplus_{\mathfrak{l} \in T}\left\langle\left(\frac{\widetilde{K}^{P} / K}{l}\right)\right\rangle .
$$

Of course, any $P$-ramified extension is $p$-primitively ramified.

Then in [65, Ch. III, $\S 2$, Theorem $2 \&$ Corollary] are characterized, for $p=2$ and $p=3$, the abelian $p$-extensions $K$ of $\mathbb{Q}$ such that $\mathscr{T}_{K, P}=1$. This is connected with the "regular kernel" of $K$ which, from results of Tate, follows similar properties which have been explained in a joint work with Jaulent [71] and developed in Jaulent-Nguyen Quang Do [72]. We can state: 
Theorem A.10. [1, Theorem III.4.2.5, Theorem IV.3.5]. Let $K$ be any number field.The following properties are equivalent:

(i) $K$ satisfies the Leopoldt conjecture at $p$ and $\mathscr{T}_{K, P}=1$;

(ii) $\mathscr{A}_{K, P}:=\mathscr{G}_{K, P}^{\mathrm{ab}}=\operatorname{Gal}\left(H_{K, P} / K\right) \simeq \mathbb{Z}_{p}^{r_{2}+1}$,

(iii) the Galois group $\mathscr{G}_{K, P}$ is a free pro-p-group on $r_{2}+1$ generators, which is equivalent to fulfill the following four conditions:

- $K$ satisfies the Leopoldt conjecture at $p$,

- $C \ell_{K} \simeq \mathbb{Z}_{p} \log _{P}\left(I_{K, P}\right) /\left(\log _{P}\left(U_{K, P}\right)+\mathbb{Q}_{p} \log _{P}\left(E_{K}\right)\right)$,

- $\operatorname{tor}_{\mathbb{Z}_{p}}\left(U_{K, P}\right)=\mu_{p}(K)$,

- $\mathbb{Z}_{p} \log _{P}\left(E_{K}\right)$ is a direct summand in $\log _{P}\left(U_{K, P}\right)$.

\section{A.5 New formalisms and use of pro- $p$-group theory}

\section{A.5.1 Infinitesimal arithmetic}

From $[69,67,73,17]$. At the same time, in his Thesis, Jaulent defines the infinitesimal arithmetic in a number field proving, in a nice conceptual framework, generalizations of our previous results, especially in the new context of logarithmic classes $[73,74]$, adding Iwasawa theory results, study of the $p$-regularity (replacing $\mathscr{T}_{K, P}$ by the tame kernel $\mathrm{K}_{2}\left(Z_{K}\right)$ of the ring of integers of $K$ ), and genus theory.

The same technical context of $\ell(=p)$-adic class field theory and a logarithmic class field theory was developed later in much papers, including computational methods of Bourbon-Jaulent [29]. He studies in [73] the logarithmic class group $\widetilde{C} \ell_{K}$ (do not confuse with $\widetilde{C \ell_{K}} P$ ) whose finiteness is equivalent to the Gross (or Gross-Kuz'min) conjecture [75], [76] (a survey is given in [1, $\S$ III.7]); see also some comments in [77, 78].

Some properties of capitulation of generalized ray class groups and of $\widetilde{C} \ell_{K}$ are given in [79, 80, 81, 82].

\section{A.5.2 Pro- $p$-group theory version}

Shortly after, at the end of the 1980's, in his thesis, Movahhedi [25, 26] gives a wide study of the abelian $p$-ramification theory, using mainly the properties of the pro- $p$-group $\mathscr{G}_{K, S}$ and deduces again most of the previous items, then he gives the main structural and cohomological properties of $\mathscr{G}_{K, P}$ and the classical characterization of the triviality of $\mathscr{T}_{K, P}$. He proposes for this to speak of " $p$-rational fields" [26, Definition 1], that is to say the number fields $K$ such that Leopoldt's conjecture holds for $p$ and $\mathscr{T}_{K, P}=1$ (cf. Theorem A.10); this was inspired by the fact that $\mathbb{Q}$ is (obviously) $p$-rational for all $p$. This vocabulary has been adopted by the arithmeticians.

Then Movahhedi gives properties of $p$-rational extensions $L / K$ and the reciprocal of our result characterizing the $p$ rationality in a $p$-extension $L / K$, in other words the "going up" of the $p$-rationality:

Theorem A.11. [25, Théorème 3, §3]. Let $L / K$ be a p-extension of number fields. The field $L$ is $p$-rational if and only if $K$ is $p$-rational and the set $T$ of tame primes, ramified in $L / K$, is p-primitive in $K$. Moreover, under these conditions, the extension $T(L)$ of $T$ to $L$ is p-primitive.

This implies that if $K$ is $p$-rational and $T p$-primitive, then any $T$-ramified $p$-extension $L / K$ fulfills the Leopoldt conjecture and $T(L)$ is $p$-primitive (a particular case was given in [50, Théorème III.4] for totally real fields).

Remark A.12. In practice, in research papers, one assumes in general an universal Leopoldt conjecture, so that the above statement becomes:

$L$ is p-rational if and only if $K$ is p-rational and $T$ is p-primitive

(equivalent to use the fixed point formula of Theorem A.5 and Corollary A.8).

In the 1990's, the classical results on $p$-ramification, $p$-rationality, and $p$-regularity about the triviality of the tame kernel $\mathrm{K}_{2}\left(Z_{K}\right)$, are amply illustrated in various directions by Movahhedi, Nguyen Quang Do, Jaulent (see Movahhedi [26], MovahhediNguyen Quang Do [70], Berger-Gras [83], Jaulent-Nguyen Quang Do [72], Jaulent-Sauzet [27] and Jaulent [17]): pro- $p$-group theory with explicit determination of a system of generators and relations for $\mathscr{G}_{K, S}$, Galois cohomology, Iwasawa's theory, Leopoldt and Gross conjectures.

Recall that in [77, Scolie, p. 112] Jaulent shows that, when $\mu_{p} \subset K$ the nullity of the $p$-Hilbert kernel $\mathrm{H}_{2}(L) \otimes \mathbb{Z}_{p}$ implies Leopoldt and Gross conjectures. Moreover [17] deals with ramification and decomposition.

Under the assumptions: $\mu_{p} \subset K, \mathrm{H}_{2}(L) \otimes \mathbb{Z}_{p}=0$, for the Hilbert kernel, and the existence of $\mathfrak{p}_{0} \in S$ such that $\mu_{K_{\mathfrak{p}_{0}}}=\mu_{K}$, some results in [84], after [25] and [70] on the primitive reciprocity laws, in the framework of $p$-rationality, describe (by means of generators and relations) the Galois group $\mathscr{G}_{K, S}$. 


\section{A.5.3 Links between these invariants and Iwasawa's theory}

Despite the fact that we limit ourselves to arithmetical invariants of the base field (which is always possible), we give a short overview on the Iwasawa context and we indicate the main references for the reader. ${ }^{4}$

The base field invariants concerned are (in the case $S=P$ ), the torsion group $\mathscr{T}_{K, P}$, the $p$-Hilbert kernel $\mathrm{H}_{2}(K) \otimes \mathbb{Z}_{p}$, and the logarithmic class group $\widetilde{C} \ell_{K}$.

Let $K_{\infty}:=K\left(\mu_{p^{\infty}}\right), \Gamma:=\operatorname{Gal}\left(K_{\infty} / K\right)=:\langle\gamma\rangle, X$ the Galois group of the maximal abelian pro- $p$-extension of $K_{\infty}$, non-ramified and in which all places totally split. For a field $k$, we put $\mu_{p^{\infty}}(k)=\mu_{p^{\infty}} \cap k^{\times}$. For any module $M$ over the Iwasawa algebra, denote by $M(i)$ the $i$ th twist on which $\Gamma$ acts by $\gamma \cdot m:=\kappa^{i}(\gamma) \cdot m^{\gamma}$, where $\kappa$ is the cyclotomic character.

Then the interpretation of the above invariants, in the Iwasawa framework is given, in part, by the following two results:

Theorem A.13. [16, Theorem 4.2]. Assuming the Leopoldt conjecture for $p$ in $K$, one has the following exact sequence $1 \rightarrow \mu_{p^{\infty}}(K) \longrightarrow \underset{\mathfrak{p} \mid p}{\bigoplus} \mu_{p^{\infty}}\left(K_{\mathfrak{p}}\right) \longrightarrow \mathscr{T}_{K, P} \longrightarrow \operatorname{Hom}_{\Gamma}\left(X, \mu_{p^{\infty}}\right) \rightarrow 1$. Then we have the following relation:

$$
\operatorname{Hom}_{\Gamma}\left(X, \mu_{p^{\infty}}\right)=\operatorname{Hom}_{\Gamma}\left(X(-1), \mathbb{Q}_{p} / \mathbb{Z}_{p}\right)=\operatorname{Hom}\left(X(-1)_{\Gamma}, \mathbb{Q}_{p} / \mathbb{Z}_{p}\right) \simeq \operatorname{Gal}\left(H_{K}^{\text {bp }} / \widetilde{K}^{P}\right)
$$

(see Remark 2.3), while (in relation with the paper of Federer-Gross-Sinnott [75]):

$$
X_{\Gamma} \simeq \widetilde{C} \ell_{K}
$$

The relation (A.8) is given in [67], then in [85, 17].

The considerable advantage of $\widetilde{C} \ell_{K}$, introduced in [73], is that it only involves some specific and explicit notions of classes and units of the base field $K$ and is then likely to be numerically calculated (Belabas-Jaulent [86]).

When $i$ varies, similar results may be interpreted by means of higher K-groups [87]. The main $\mathrm{K}_{2}$-theoretic interpretation is given as follows:

Theorem A.14. [16, Theorem 5.6]. One has: $\left(\mathrm{H}_{2}(K) \otimes \mathbb{Z}_{p}\right)^{*}=\operatorname{Ker}_{P}^{2}\left(\mathbb{Q}_{p} / \mathbb{Z}_{p}(-1)\right)$; if $K$ contains $\mu_{p^{e}}, e \geq 1$, one obtains the perfect duality: $\operatorname{Gal}\left(H_{K}^{\mathrm{bp}} / \widetilde{K}^{P}\right)\left[p^{e}\right] \times\left(\mathrm{H}_{2}(K) / p^{e} \mathrm{H}_{2}(K)\right)(-1) \longrightarrow \mu_{p^{e}}$, where $T\left[p^{e}\right]:=\left\{x \in T, p^{e} \cdot x=0\right\}$ for a $\mathbb{Z}_{p}$-module $T$, and where $\operatorname{Ker}_{P}^{2}$ is the kernel of the localization homomorphism $\mathrm{H}_{2}\left(\mathscr{G}_{K, P}\right) \longrightarrow \underset{\mathfrak{p} \mid p}{\bigoplus} \mathrm{H}_{2}\left(\mathscr{G}_{K, \mathfrak{p}}\right)$.

This result of duality does appear in [67, 85]. If $\mu_{p} \subset K$, the nullity of $\mathrm{H}_{2}(K) \otimes \mathbb{Z}_{p}$ is equivalent to that of $\widetilde{C} \ell_{K}$, which makes the link with the above Scolie [77, Scolie, p. 112] of Jaulent. For relations between logarithmic classes and higher K-groups, mention the work of Jaulent-Michel [88] and that of Hutchinson [89].

\section{A.5.4 Synthesis 2003-2005}

Because our Crelle papers, were written in french, whence largely ignored, all the results and consequences, that we have given in $[61,50,63,64,66,65,51]$, were widely developed and improved in [1] where a systematic and general use of ramification and decomposition is considered, the infinite places playing a specific role (decomposition or complexification).

Furthermore, [1, Theorem V.2.4 and Corollary V.2.4.2] give a characterization (with explicit governing fields) of the existence of degree $p$ cyclic extensions of $K$ with given ramification and decomposition. This criteria has been used by Hajir-Maire and Hajir-Maire-Ramakrishna in several of their papers for results on $S$-ramified pro- $p$-groups (see, e.g., [90, Theorem 5.3], [91, Remark 2.2.] for the most recent publications).

\section{A.6 Present theoretical and algorithmic aspects}

One may say that there is no important progress for $p$-rationality, for itself, since $p$-rational fields are in some sense the "simplest fields" in a $p$-adic sense, but that the significance of the $p$-adic properties of the groups $\mathscr{T}_{K, S}$, in much domains of number theory, has given a great lot of heuristics, conjectures, computations; so we shall now describe some of these aspects with some illustrations (it is not possible to be comprehensive since the concerned literature becomes enormous).

\footnotetext{
${ }^{4}$ This Subsection, describing the two different (but equivalent) techniques, is close to personal communications of Jean-François Jaulent and Thong Nguyen Quang Do (up to the notations and some comments). We thank them also for some remarks and corrections about this subsection.
} 


\section{A.6.1 Absolute abelian Galois group $A_{K}$ of $K$}

Let $K^{\mathrm{ab}}$ be the maximal abelian pro-extension of $K$. In [92], [93], Angelakis-Stevenhagen and Angelakis, after some work by Kubota [48] and Onabe [94], provide a direct computation of the profinite group $A_{K}:=\operatorname{Gal}\left(K^{\mathrm{ab}} / K\right)$ for imaginary quadratic fields $K$, and use it to obtain many different $K$ that all have the same "minimal" absolute abelian Galois group, which is in some sense a condition of minimality of the groups $\mathscr{T}_{K, P}$ for all primes $p$. They obtain for instance, among other results and numerical illustrations:

Theorem A.15. [92, Theorem 4.1 \& Section 7]. An imaginary quadratic field $K \neq \mathbb{Q}(i), \mathbb{Q}(\sqrt{-2})$ of class number 1 has absolute abelian Galois group isomorphic to $\widehat{\mathbb{Z}}^{2} \times \prod_{n \geq 1} \mathbb{Z} / n \mathbb{Z}$.

This corresponds to the fact that such fields are $p$-rational for all $p$ (up to the factors $\mathscr{W}_{K, P}$ for $p=2,3$ ). Then the generalization to an arbitrary $K$ involves the $\mathscr{T}_{K, P}$ for all primes $p$, giving:

Theorem A.16. [95, Theorem 2.1 \& Corollary 2.1]. Let $K^{\mathrm{ab}}$ be the maximal Abelian pro-extension of $K$. Let $\mathscr{H}_{K}$ be the compositum, over $p$, of the maximal P-ramified Abelian pro-p-extensions $H_{K, P}$ of $K$. Under the Leopoldt conjecture, there exists an Abelian extension $L_{K}$ of $K$ such that $\mathrm{Gal}\left(L_{K} / K\right) \simeq \prod_{p} \mathscr{T}_{K, P}$ and such that $\mathscr{H}_{K}$ is the direct compositum over $K$ of $L_{K}$ and the maximal $\widehat{\mathbb{Z}}$-extension of $K$, and such that we have the non-canonical isomorphism (for some explicit integers $\delta$ and $w$ ):

$$
\operatorname{Gal}\left(K^{\mathrm{ab}} / L_{K}\right)=\widehat{\mathbb{Z}}^{r_{2}+1} \times \operatorname{Gal}\left(K^{\mathrm{ab}} / \mathscr{H}_{K}\right) \simeq \widehat{\mathbb{Z}}^{r_{2}+1} \times \prod_{n \geq 1}\left((\mathbb{Z} / 2 \mathbb{Z})^{\delta} \times \mathbb{Z} / w n \mathbb{Z}\right)
$$

Angelakis-Stevenhagen conjecture in [92, Conjecture 7.1] the infiniteness of imaginary quadratic fields $K$ such that $A_{K} \simeq \widehat{\mathbb{Z}}^{2} \times \prod_{n \geq 1} \mathbb{Z} / n \mathbb{Z}$.

Note that when the $p$-class group of $K$ is non-trivial, $K$ is $p$-rational if and only if $C \ell_{K}$ is cyclic and the $p$-Hilbert class field $H_{K}$ is contained in $\widetilde{K}^{P}$ (assuming $\mathscr{W}_{K, P}=1$ ).

Whence the importance of fields $K$ being $p$-rational for all $p$ (or more precisely such that $\mathscr{T}_{K, P}=\mathscr{W}_{K, P}$ for all $p$ ); it is an easier problem only for $\mathbb{Q}$ and imaginary quadratic fields, but dreadfully difficult when $K$ contains units of infinite order since it is an analogous question as for Fermat's quotients of algebraic numbers (various heuristics and conjectures in [96]), or values of $L$-functions which intervene as in Coates-Li [33, 34], Goren [97], and more or less, in many papers as Boeckle-Guiraud-Kalyanswamy-Khare [98] when the normalized $p$-adic regulator is a unit. We have conjectured that, in any given number field $K, \mathscr{T}_{K, P}=1$ for almost all $p$.

\section{A.6.2 Greenberg's conjecture on Iwasawa's $\lambda, \mu$}

For a totally real number field $K$, consider (under the Leopoldt conjecture) the cyclotomic $\mathbb{Z}_{p}$-extension $K^{\mathrm{c}}$ of $K$. Then Greenberg has conjectured in [99] that the Iwasawa's invariants $\lambda$ and $\mu$ are zero.

Equivalent formulations of this conjecture have been given, as in [100] for an encompassing approach covering the necessary and sufficient conditions considered by Greenberg in two particular cases (we give up to provide a complete bibliography), but we must mention that the two invariants $\mathscr{T}_{K, P}$ and $\widetilde{C} \ell_{K}$ (the logarithmic class group of Jaulent) are in some sense "governing invariants" for the Greenberg conjecture (in a theoretical and numerical viewpoint) and explain the $p$-adic obstructions for a standard proof in the framework of Iwasawa's theory; for instance, as soon as $\mathscr{T}_{K, P}=1$ or $C \ell_{K}=1$, Greenberg's conjecture is true for trivial reasons. For this, see [101, Théorèmes 3.4, 4.8, 6.3] and [102] about $\mathscr{T}_{K, P}$, then the interpretation by Jaulent with the group of universal norms [103] and the following criterion (under the Gross-Leopoldt conjecture):

Theorem A.17. [104, Théorème 7, § 1.4]. The totally real number field K fulfills the conjecture of Greenberg if and only if its logarithmic class group $\widetilde{C} \ell_{K}$ capitulates in the cyclotomic $\mathbb{Z}_{p}$-extension $K^{\mathrm{c}}$ of $K$.

If Greenberg's conjecture is true (which is no doubt), such general condition of capitulation is very reassuring since we recall that, on the contrary, the group $\mathscr{T}_{K, P}$ never capitulates. Moreover the property of capitulation (well known in Hilbert's class fields) is more general for generalized ray class groups and, especially, is possible in absolute abelian extensions as shown in many papers including [105], Bosca [106], then [81, 82].

This result may be deduced in the framework of Iwasawa's theory recalled in the $\S$ A.5.3 [100, Théorème 2.1].

Unfortunately, at the time of writing this text, no proof of Greenberg's conjecture does exist, despite some unsuccessful attempts in [107, 108] (to understand the key-points of the $p$-adic obstruction to be analyzed and possibly completed, see [104, $\S 3.4$, Remark] and [102, § 6.2, Diagram]). 


\section{A.6.3 Galois representations with open image}

For constructions by Greenberg, in [109], of continuous Galois representations $\operatorname{Gal}(\overline{\mathbb{Q}} / \mathbb{Q}) \rightarrow \mathrm{GL}_{n}\left(\mathbb{Z}_{p}\right)$ with open image, the $p$-rational fields play a great role, and the first obvious case is that of $p$-regular cyclotomic fields $\mathbb{Q}\left(\mu_{p}\right)$ which are $p$-rational (yet reported by [3], [65], and generalized by introducing in [71] the notion of $p$-regularity of number fields that we do not develop in this paper, for short, but which behaves as $p$-rationality; see a survey in [72]).

Then, an interesting typical conjecture is the following:

Conjecture A.18. [109, Conjecture 4.2.1]. For any odd prime $p$ and for any $t \geq 1$, there exists a p-rational field $K$ such that $\operatorname{Gal}(K / \mathbb{Q}) \simeq(\mathbb{Z} / 2 \mathbb{Z})^{t}$

Numerical examples and statistics have been given for various $p$ and $t$; see [109] and (Robert Bradshaw) the 3-rationality of:

$$
K=\mathbb{Q}(\sqrt{13}, \sqrt{145}, \sqrt{209}, \sqrt{269}, \sqrt{373}, \sqrt{-1})
$$

Some PARI/GP programs are given in Pitoun-Varescon [110, 111], and [20, §5.2] showing the 3-rationality of:

$$
K=\mathbb{Q}(\sqrt{-2}, \sqrt{-5}, \sqrt{7}, \sqrt{17}, \sqrt{-19}, \sqrt{59})
$$

For fixed $p$ (e.g., $p=3$ ), the probability of $p$-rationality decreases dramatically when $t \rightarrow \infty$; indeed, if $\operatorname{Gal}(K / \mathbb{Q}) \simeq(\mathbb{Z} / 2 \mathbb{Z})^{t}$, $K$ is $p$-rational if and only if the $2^{t}-1$ quadratic subfields $k$ of $K$ are $p$-rational whose probability is of the order of $\left(\frac{1}{p}\right)^{2^{t}-1}$ assuming that class groups and units of each $k$ are random and largely independent regarding the $p$-adic properties.

\section{A.6.4 Order of magnitude of $\mathscr{T}_{K, P}$ and conjectures}

We have conjectured in [96, Conjecture 8.11] that for a fixed number field $K, \mathscr{T}_{K, P}=1$ for all $p \gg 0$. Moreover, all numerical calculations show that the non- $p$-rationality constitutes an exception.

In another direction, fixing $p$ and taking $K$ in some given infinite family $\mathscr{K}$ (e.g., real fields of given degree $d$ ) we have given extensive numerical computations in direction of the following " $p$-adic Brauer-Siegel” property:

Conjecture A.19. [30, Conjecture 8.1]. There exists a constant $\mathscr{C}_{p}(\mathscr{K})$ such that:

$$
v_{p}\left(\# \mathscr{T}_{K, p}\right) \leq \mathscr{C}_{p}(\mathscr{K}) \cdot \frac{\log _{\infty}\left(\sqrt{D_{K}}\right)}{\log _{\infty}(p)}
$$

for all $K \in \mathscr{K}$, where $\log _{\infty}$ is the usual complex logarithm.

Thus there are two questions about $C_{p}(K):=\frac{v_{p}\left(\# \mathscr{T}_{K, P}\right) \cdot \log _{\infty}(p)}{\log _{\infty}\left(\sqrt{D_{K}}\right)}$ and the quantities $\mathscr{C}_{p}:=\sup _{K}\left(C_{p}(K)\right), \mathscr{C}_{K}:=\sup _{p}\left(C_{p}(K)\right)$ :

(i) The existence of $\mathscr{C}_{K}<\infty$, for a given $K$, only says that the Conjecture " $\mathscr{T}_{K, P}=1 \forall p \gg 0$ " is true for the field $K$; for this field, we get $\limsup _{p}\left(C_{p}(K)\right)=0$. A.19).

(ii) If $\mathscr{C}_{p}<\infty$ does exist for a given $p$, we have an universal $p$-adic analog of Brauer-Siegel theorem (the above Conjecture

These questions being out of reach, many results give, on the contrary, the infintness of primes $p$ yielding the $p$-rationality of a field $K$, in general under the $a b c$ conjecture, following the method given by Silverman [112], Graves-Murty [113], Boeckle-Guiraud-Kalyanswamy-Khare [98], Maire-Rougnant [114]; for instance:

Theorem A.20. [114, Corollary to Theorem A]. Let $K$ be a real quadratic field or an imaginary $S_{3}$-extension. If the generalized abc-conjecture holds for $K$, then $\#\{p \leq x, K$ is $p$-rational $\} \geq c \cdot \log (x)$ as $x \rightarrow \infty$, for some constant $c>0$ depending on $K$.

This shows the awesome distance between the two aspects of the problem; indeed, for $K=\mathbb{Q}(\sqrt{5})$, no prime number (up to $p<10^{14}$ from Elsenhans-Jahnel: https : / / oeis.org/A060305) is known giving $\mathscr{T}_{K, P} \neq 1$.

In another viewpoint, as in [115] (after some works of Hartung, Horie, Naito) and [116], it is shown, using modular forms, the infiniteness of $p$-rational real quadratic fields for $p=3$ and $p=5$. 


\section{A.6.5 Fermat curves}

To study Fermat curves of exponent $p$, one uses the base field $K=\mathbb{Q}\left(\mu_{p}\right)$ and works in some Kummer extensions; for instance:

(i) Shu [117] gives general formulae of the root numbers of the Jacobian varieties of the Fermat curves $X^{p}+Y^{p}=\delta$, where $\delta$ is an integer, and studies their distribution. In this article the Vandiver conjecture or the regularity of $p$ implies some precise properties of the Selmer groups of these Jacobian varieties.

(ii) Davis-Pries [118] work in $P$-ramified Kummer extensions of $K$ with $P=\left\{\mathfrak{p}=\left(1-\zeta_{p}\right)\right\}$, as follows. Let $L \subset H_{K, P}$ be defined by:

$$
L=K\left(\sqrt[p]{\zeta_{p}}, \sqrt[p]{1-\zeta_{p}}, \cdots, \sqrt[p]{1-\zeta_{p}^{r}}\right), r=\frac{p-1}{2}
$$

The Kummer radical of $L$ is also generated by the real cyclotomic units and the numbers $\zeta_{p}, 1-\zeta_{p}$. In the same way as previously, non-Vandiver's conjecture or non-regularity for $p$ are crucial obstructions.

Under the Vandiver conjecture, this radical is of $p$-rank $r+1$ since it is then given by $E_{K} \cdot\left\langle 1-\zeta_{p}\right\rangle$ modulo $K^{\times p}$.

Under the regularity of $p$, we get $\mathscr{T}_{K, P}=1$ (reflection theorem (A.3)) and $L$ is the maximal $p$-elementary subextension of $H_{K, P} ; L / K$ being $p$-ramified, whence $p$-primitively ramified ( $\S$ A.4.3), this gives the $p$-rationality of $L$.

Let $E$ be the maximal $p$-elementary subextension of $H_{L, P}$; since $\mathscr{T}_{L, P}=1$ with $E / L p$-ramified, we then have $\mathscr{T}_{E, P}=1$ and $\operatorname{rk}_{p}(\operatorname{Gal}(E / L))=r \cdot p^{r+1}+1$. One can deduce that $C \ell_{L}=C \ell_{E}=1$ since $E / K$ is totally ramified at $\mathfrak{p}$ (Theorem A.1 and Chevalley's formula in any successive $p$-cyclic extensions in $E / K)$.

In simple cases as $p=37$, where ${ } C \ell_{K}=p$ and where $H_{K} \subseteq L$ in which $p$ splits, the formula of Theorem A.1 gives $\operatorname{rk}_{p}\left(\mathscr{T}_{L, P}\right)=\operatorname{rk}_{p}\left(C \ell_{L}^{P}\right)+p-1$, whence $\operatorname{rk}_{p}(\operatorname{Gal}(E / L))=r \cdot p^{r+1}+2 r+1+\operatorname{rk}_{p}\left(C \ell_{L}^{P}\right)$ depending on $C \ell_{L}^{P}$, a priori unknown.

The purpose of [118] is to get information on $\mathrm{H}^{1}(\mathrm{Gal}(E / K), M)$ for some $\mathrm{Gal}(E / K)$-modules $M$, subquotients of the relative homology $H_{1}\left(U, Y ; \mathbb{F}_{p}\right)$ of the Fermat curve, where $U$ is the affine curve $x^{p}+y^{p}=1$ and $Y$ the set of $2 p$ cusps where $x y=0$. They completely elucidate the case $p=3$.

\section{A.7 Computational references and numerical tables}

Many references may be cited:

The first table for the computation of $\# \mathscr{T}_{K, P}$ for imaginary quadratic fields is that of Charifi [119], using formula (A.6). In Hatada $[120,121]$ the computations correspond to statistics about the values (modulo $p$ ) of the normalized regulator $\mathscr{R}_{K, P}$ of real fields as $K=\mathbb{Q}(\sqrt{5})$ by the way of Fibonacci numbers and values at $2-p$ of zeta-functions as we have mentioned in $\S$ A.3.2. He obtains for instance that $\mathbb{Q}(\sqrt{2})$ is $p$-rational for all $p \leq 20000$, except $p=13,31$ (our program gives the next exception $p=1546463$ up to $10^{8}$ ).

A precise study of $p$-rationality of imaginary quadratic fields is given by Angelakis-Stevenhagen in [92, Section 7].

A wide study of $\mathscr{T}_{K, P}$, with tables and publication of PARI/GP programs, is done by Pitoun [110, Chapitre 4], but these more conceptual programs are not so easy to manage by the reader. Then some statistical results with tables are given by Pitoun-Varescon in [111].

In [122] Hofmann-Zhang compute the valuation of the (usual) $p$-adic regulators of cyclic cubic fields with discriminant up to $10^{16}$, for $3 \leq p \leq 100$, and observe the distribution of these valuations.

About the conjecture of Greenberg [109] Kraft-Schoof [123] have computed such Iwasawa's invariants and confirm the conjecture for $p=3$ and conductors $f$ of real quadratic fields $f \neq 1(\bmod 3)$ up to $10^{4}$. In [20], some heuristics on the conjecture and numerical examples are given with programs; then we illustrate the following conjecture of Hajir-Maire [43, Conjecture 4.16]:

Given a prime $p$ and an integer $m \geq 1$, co-prime to $p$, there exist a totally imaginary field $K_{0}$ and a degree $m$ cyclic extension $K / K_{0}$ such that $K$ is p-rational; it is conjectured that the statement is true taking for $K_{0}$ an imaginary p-rational quadratic field.

In [124, Table 1, $\S 2]$, Barbulescu-Ray give explicit $p$-rational large compositum of quadratic fields. We may cite some works by Bouazzaoui [125], El Habibi-Ziane [47] based on $p$-rationality of quadratic fields.

In the similar context of $p$-ramification, a new PARI/GP package allows the computation of the logarithmic class group $\widetilde{C} \ell_{K}$ of a number field by Belabas-Jaulent [86] that we can illustrate as follows where the invariants $[Y, X, Z]$ are linked by the exact sequence:

$$
1 \rightarrow X \longrightarrow Y:=\widetilde{C} \ell_{K} \longrightarrow Z:=C \ell_{K}^{P}:=C \ell_{K} /\left\langle c \ell_{K}(P)\right\rangle \rightarrow 1
$$




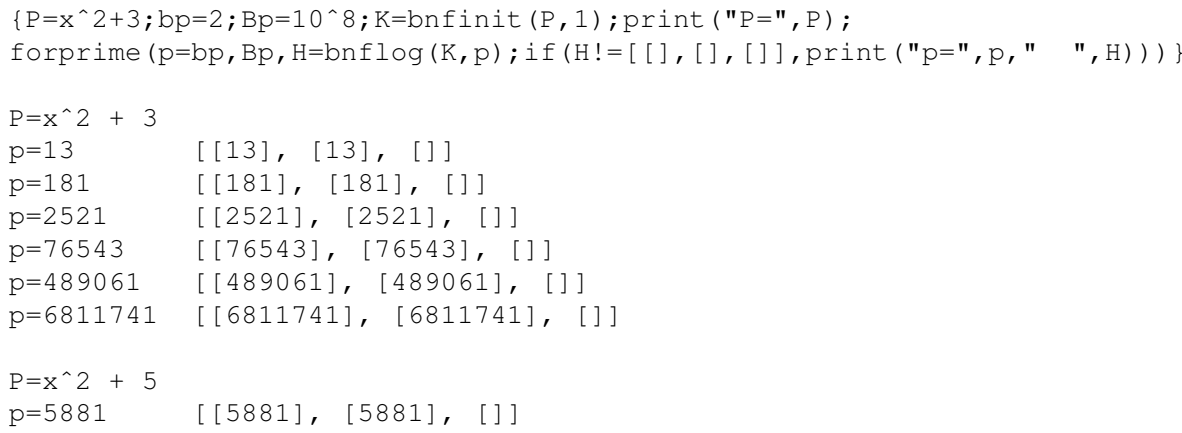

These are the only solutions for $p<10^{8}$. More computations would give heuristics to see if the analogous conjecture: " $\widetilde{C} \ell_{K}=1$ for all $p \gg 0$ ", is credible or not since the rarefaction of non-trivial cases is similar to that of the groups $\mathscr{T}_{K, P}$.

The case of real quadratic fields is studied in $[101, \S 5.2]$ with a table and in $[104, \S 2.4]$, after the work of Ozaki-Taya [126] and others.

In another direction, the paper [127] of Maire-Rougnant gives examples of triviality of isotopic components of the torsion groups $\mathscr{T}_{K, P}$; more precisely the fields $K$ are cyclic extensions of $\mathbb{Q}$ of degrees 3 and 4 (from polynomials of Balady, Lecacheux, Balady-Washington) and $S_{3}$-extensions of $\mathbb{Q}$.

In [30], are given numerous programs to test some heuristics and conjectures about the order of magnitude of the groups $\mathscr{T}_{K, P}$ in totally real number fields in a Brauer-Siegel framework.

\section{A.8 Conclusion and open questions}

In all the aspects of $p$-rationality that we have developed (theoretical and computational), some interesting applications are done today, including for instance, for the most recent ones, [43] by Hajir-Maire on the $\mu$-invariant in Iwasawa's theory, then [90] by Hajir-Maire-Ramakrishna, showing the existence of $p$-rational fields having large $p$-rank of the class group, or [91] about the existence of a solvable number field $L, P$-ramified, whose $p$-Hilbert class field tower is infinite. See the bibliographies of these articles to expand the list of contributions.

Of course it is not possible to evoke all the studied families of pro- $p$-groups having some logical links with $S$-ramification (with more general sets $S$ regarding $P$ ) as, for instance, that of "mild groups" introduced by Labute [12] (and [13] for the case $p=2$ ) dealing with the numbers of generators $d(G)$ and of relations $r(G)$ and defined as follows:

A class of finitely presented pro-p-groups $G$ of cohomological dimension 2 such that $r(G) \geq d(G)$ and $d(G) \geq 2$ arbitrary.

Many articles where then published giving an overview of the wide variety of such groups as the following short excerpt of a result of Schmidt about global fields [46, Theorem 1.1]:

Let $S, T, \mathscr{M}$ be pairwise disjoint sets of places of $K$, where $S$ and $T$ are finite and $\mathscr{M}$ has Dirichlet density 0. Then there exists a finite set of places $S_{0}$ of $K$ which is disjoint from $S \cup T \cup \mathscr{M}$ and such that the group $\mathscr{G}_{K, S \cup S_{0}}^{T}$ has cohomological dimension 2.

But let's go back to the basic abelian invariants, asking some open questions:

(i) We know the fixed point formula in a $p$-extension $L / K$ (under the conjecture of Leopoldt), but, even in a $p$-cyclic extension with Galois group $G$, and contrary to the case of $p$-class groups (as done in [128] after a very long history), we do not know how to compute the filtration $\left(M_{i}\right)_{i \geq 0}$, of $M:=\mathscr{T}_{K, P}$, defined inductively by:

$$
M_{0}=1 \text { and } M_{i+1} / M_{i}:=\left(M / M_{i}\right)^{G} \text {, for all } i \geq 0 .
$$

(ii) The explicit computation of the $p$-rank, $\widetilde{r}_{K, S}(1.3)$, of $\mathscr{A}_{K, S} / \mathscr{T}_{K, S}$ for $S \subseteq P$, is available only in favorable Galois cases with an algebraic reasoning on the canonical representation $\mathbb{Q}_{p} \log _{S}\left(E_{K}\right)$ given by the Herbrand theorem on units under Leopoldt's conjecture (see $\S 2.4$ ).

(iii) In the definition of $\mathscr{W}_{K, S}:=W_{K, S} / \operatorname{tor}_{\mathbb{Z}_{p}}\left(\bar{E}_{K}^{S}\right)$, we do not know how to compute $\operatorname{tor}_{\mathbb{Z}_{p}}\left(\bar{E}_{K}^{S}\right) \supseteq \imath_{S}\left(\mu_{K}\right)$ when $S \subsetneq P$. We ignore, in a $p$-adic framework, if Leopoldt's conjecture is sufficient to obtain the responses apart from a Galois context.

A reasonable conjecture is that $\operatorname{tor}_{\mathbb{Z}_{p}}\left(\bar{E}_{K}^{S}\right)=l_{S}\left(\mu_{K}\right)$ whatever $K, p$ and $S$; but this must be deepened.

We hope that our programs in $\S 3.1 .1$ may help to give heuristics about this. 


\section{Note}

In the programs in verbatim text, one must replace the symbol of power (in a^b) by the corresponding PARI/GP symbol (which is nothing else than that of the computer keyboard); otherwise the program does not work (this is due to the character font used by some Journals). The good print for the programs is also available at:

https://www.dropbox.com/s/1srmksbr2ujf40i/Incomplete\%20p-ramification.pdf?dl=0

\section{Acknowledgments}

I would like to thank Christian Maire and Jean-François Jaulent for fruitful discussions and information concerning some aspects of pro- $p$-groups and $S$-ramification.

I warmly thank the referees for the care reading of the paper and their comments.

\section{References}

[1] G. Gras, Class Field Theory: From Theory to Practice, corr. 2nd ed., Springer Monographs in Mathematics, Springer, 2005, xiii+507 pages.

[2] J-P. Serre, Cohomologie galoisienne, Lect. Notes in Math. 5, Springer-Verlag 1964, cinquième édition 1991; English translation: Galois cohomology, Springer 1997; corrected second printing: Springer Monographs in Math., 2002.

[3] I. R. Šafarevič, Extensions with given points of ramification, Publ. Math. Inst. Hautes Etudes Sci., 18 (1964), 71-95; American Math. Soc. Transl., Ser. 2, 59 (1966), 128-149.

[4] A. Brumer, Galois groups of extensions of algebraic number fields with given ramification, Michigan Math. J., 13 (1966), 33-40.

[5] J. Neukirch, A. Schmidt, K. Wingberg, Cohomology of Number Fields, Springer-Verlag (2000), 2nd edition, Grundlehren der Math. Wissenschaften 323, Springer-Verlag, 2008.

[6] K. Wingberg, On Galois groups of p-closed algebraic number fields with restricted ramification, J. Reine Angew. Math., 400 (1989), 185-202.

[7] K. Wingberg, On Galois groups of p-closed algebraic number fields with restricted ramification II, J. Reine Angew. Math., 416 (1991), 187-194.

[8] M. Yamagishi, A note on free pro-p-extensions of algebraic number fields, J. Théor. Nombres Bordeaux, 5(1) (1993), $165-178$.

[9] C. Maire, On the $\mathbb{Z}_{\ell}$-rank of abelian extensions with restricted ramification, J. Number Theory, 92 (2002), $376-404$.

[10] C. Maire, On the $\mathbb{Z}_{\ell}$-rank of abelian extensions with restricted ramification (addendum), J. Number Theory, 98 (2003), 217-220.

[11] C. Maire, Sur la dimension cohomologique des pro-p-extensions des corps de nombres, J. Théor. Nombres Bordeaux, 17(2) (2005), 575-606.

[12] J. Labute, Mild pro-p-groups and Galois groups of p-extensions of $\mathbb{Q}$, J. Reine Angew. Math., 596 (2006), $155-182$.

[13] J. Labute, J. Mináč, Mild pro-2-groups and 2-extensions of $\mathbb{Q}$ with restricted ramification, J. Algebra, 332(1) (2011), $136-158$.

[14] D. Vogel, p-extensions with restricted ramification - The mixed case (2007), available at https: / / epub.uni-regensburg.de/13820/

[15] O. Neumann, On p-closed number fields and an analogue of Riemann's existence theorem. Algebraic number fields: L-functions and Galois properties, Proc. Sympos., Univ. Durham (1975), pp. 625-647. Academic Press, London, 1977.

[16] T. Nguyen Quang Do, Sur la $\mathbb{Z}_{p}$-torsion de certains modules galoisiens, Ann. Inst. Fourier, 36(2) (1986), $27-46$.

[17] J-F. Jaulent, Théorie $\ell$-adique globale du corps de classes, J. Théor. Nombres Bordeaux, 10(2) (1998), $355-397$.

[18] F. Bertrandias, J-J. Payan, Г-extensions et invariants cyclotomiques, Ann. Sci. Ec. Norm. Sup. 4e série, 5(4) (1972), $517-548$.

[19] G. Gras, The p-adic Kummer-Leopoldt Constant: Normalized p-adic Regulator, Int. J. Number Theory, 14(2) (2018), $329-337$. 
[20] G. Gras, On p-rationality of number fields. Applications-PARI/GP programs, Pub. Math. Besançon (Théorie des Nombres), Années 2018/2019 (2019), arxiv:1709.06388 [math.NT]

[21] I. B. Fesenko, S. V. Vostokov, Local Fields and Their Extensions, American Math. Soc., Translations of Math. Monographs, 121, Second Edition, 2002.

[22] J-F. Jaulent, Sur l'indépendance $\ell$-adique de nombres algébriques, J. Number Theory, 20 (1985), $149-158$.

[23] D. Nelson, A variation on Leopoldt's conjecture: some local units instead of all local units (2013), arxiv:1308.4637 [math.NT]

[24] The PARI Group -PARI/GP, version 2.9. 0, Université de Bordeaux (2016).

[25] A. Movahhedi, Sur les $p$-extensions des corps $p$-rationnels, Thèse, Univ. Paris VII, 1988.

[26] A. Movahhedi, Sur les p-extensions des corps p-rationnels, Math. Nachr., 149 (1990), 163-176.

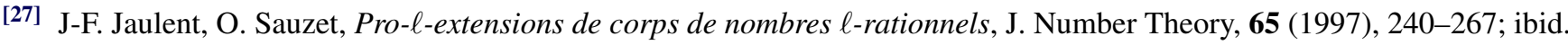
80 (2000), 318-319.

[28] J-F. Jaulent, O. Sauzet, Extensions quadratiques 2-birationnelles de corps de nombres totalement réels, Pub. Matemàtiques, 44 (2000), 343-351.

[29] C. Bourbon, J-F. Jaulent, Propagation de la 2-birationalité, Acta Arithmetica, 160 (2013), 285-301.

[30] G. Gras, Heuristics and conjectures in the direction of a p-adic Brauer-Siegel theorem, Math. Comp., 88(318) (2019), 1929-1965.

[31] E. Lecouturier, On the Galois structure of the class group of certain Kummer extensions, J. London Math. Soc. (2), 98 (2018), 35-58.

[32] K. Schaefer, E. Stubley, Class groups of Kummer extensions via cup products in Galois cohomology, Trans. Amer. Math. Soc., Published electronically: May 30, 2019

[33] J. Coates, Y. Li, Non-vanishing theorems for central L-values of some elliptic curves with complex multiplication (2018), (in press).

[34] J. Coates, Y. Li, Non-vanishing theorems for central L-values of some elliptic curves with complex multiplication II (2019), (in press).

[35] T. Nguyen Quang Do, Sur la structure galoisienne des corps locaux et la théorie d'Iwasawa, Compositio Math., 46(1) (1982), 85-119.

[36] H. Koch, Galois theory of p-extensions (English translation of "Galoissche Theorie der p-Erweiterungen”, 1970), Springer Monographs in Math., Springer, 2002.

[37] F. Hajir, C. Maire, Tamely ramified towers and discriminant bounds for number fields, Compositio Math., 128(1) (2001), 35-53.

[38] F. Hajir, C. Maire, Tamely ramified towers and discriminant bounds for number fields II, J. of Symbolic Computation, 33(4) (2002), 415-423.

[39] F. Hajir, C. Maire, Extensions of number fields with wild ramification of bounded depth, International Mathematics Research Notices, 13 (2002), 667-696.

[40] C. Maire, Cohomology of number fields and analytic pro-p-groups, Mosc. Math. J., 10(2) (2010), 399-414, 479.

[41] C. Maire, On the quotients of the maximal unramified 2-extension of a number field, Documenta Mathematica, 23 (2018), 1263-1290.

[42] F. Hajir, C. Maire, Analytic Lie extensions of number fields with cyclic fixed points and tame ramification (2018), (in press).

[43] F. Hajir, C. Maire, Prime decomposition and the Iwasawa mu-invariant, Math. Proc. Camb. Phil. Soc., 166 (2019), 599-617. Published online: 26 April 2018.

[44] O. Neumann, On p-closed algebraic number fields with restricted ramification, Izv. Akad. Nauk USSR, ser. Math. 39, 2 (1975), 259-271; English translation: Math. USSR, Izv., 9 (1976), 243-254.

[45] K. Haberland, Galois cohomology of algebraic number fields. With two appendices by Helmut Koch, Thomas Zink, V.E.B. Deutscher Verlag der Wissenschaften, 1978.

[46] A. Schmidt, Über pro-p-fundamentalgruppen markierter arithmetischer kurven, J. Reine Angew. Math., 640 (2010), 203-235. 
[47] A. El Habibi, M. Ziane, p-Rational Fields and the Structure of Some Modules, (2018), arxiv:1804.10165 [math.NT].

[48] T. Kubota, Galois group of the maximal abelian extension of an algebraic number field, Nagoya Math. J., 12 (1957), 177-189.

[49] H. Miki, On the maximal abelian $\ell$-extension of a finite algebraic number field with given ramification, Nagoya Math. J., 70 (1978), 183-202.

[50] G. Gras, Groupe de Galois de la p-extension abélienne p-ramifiée maximale d'un corps de nombres, J. Reine Angew. Math., 333 (1982), 86-132, doi: 10.1515/crll.1982.333.86

[51] G. Gras, Théorèmes de réflexion, J. Théor. Nombres Bordeaux, 10(2) (1998), 399-499.

[52] G. Gras, New criteria for Vandiver's conjecture using Gauss sums. Heuristics and numerical experiments (2019), arxiv:1808.03443 [math.NT]

[53] J. S. Ellenberg, A. Venkatesh, Reflection principles and bounds for class group torsion, Int. Math. Res. Not., 2007(1) (2007).

[54] G. Gras, Annihilation of $\operatorname{tor}_{\mathbb{Z}_{p}}\left(\mathscr{G}_{K, S}^{\mathrm{ab}}\right)$ for real abelian extensions $K / \mathbb{Q}$, Communications in Advanced Mathematical Sciences, 1(1) (2018), 5-34.

[55] B. Oriat, Lien algébrique entre les deux facteurs de la formule analytique du nombre de classes dans les corps abéliens, Acta Arithmetica, 46 (1986), 331-354.

[56] Y. Amice, J. Fresnel, Fonctions zêta p-adiques des corps de nombres abéliens réels, Acta Arithmetica, 20(4) (1972), 353-384.

[57] J. Coates, p-adic L-functions and Iwasawa's theory, In: Proc. of Durham Symposium 1975, New York-London, (1977), 269-353.

[58] J-P. Serre, Sur le résidu de la fonction zêta p-adique d'un corps de nombres, C.R. Acad. Sci. Paris, 287(Série I) (1978), $183-188$.

[59] P. Colmez, Résidu en $s=1$ des fonctions zêta p-adiques, Invent. Math., 91 (1988), 371-389.

[60] J. Assim, A. Movahhedi, Galois codescent for motivic tame kernels (2019), (in press).

[61] G. Gras, Étude d'invariants relatifs aux groupes des classes des corps abéliens, Journées Arithmétiques de Caen (Univ. Caen, Caen, 1976), Astérisque, No. 41-42, Soc. Math. France, Paris (1977), pp. 35-53

[62] K. A. Ribet, Bernoulli numbers and ideal classes, Gaz. Math., 118 (2008), 42-49.

[63] G. Gras, Logarithme p-adique et groupes de Galois, J. Reine Angew. Math., 343 (1983), 64-80.

[64] G. Gras, Sur la p-ramification abélienne, Conférence donnée à l'University Laval, Québec, Mathematical series of the department of mathematics, 20 (1984), 1-26.

[65] G. Gras, Remarks on $K_{2}$ of number fields, J. Number Theory, 23(3) (1986), 322-335.

[66] G. Gras, Plongements kummériens dans les $\mathbb{Z}_{p}$-extensions, Compositio Math., 55(3) (1985), 383-396.

[67] J-F. Jaulent, L'arithmétique des $\ell$-extensions Thèse de doctorat d'Etat, Pub. Math. Besançon (Théorie des Nombres) (1986), 1-349.

[68] C. Chevalley, Sur la théorie du corps de classes dans les corps finis et les corps locaux, Thèse no. 155, Jour. of the Faculty of Sciences Tokyo, 2 (1933), 365-476.

[69] J-F. Jaulent, S-classes infinitésimales d'un corps de nombres algébriques, Ann. Sci. Inst. Fourier, 34(2) (1984), 1-27.

[70] A. Movahhedi, T. Nguyen Quang Do, Sur l'arithmétique des corps de nombres p-rationnels, Séminaire de Théorie des Nombres, Paris 1987-88, Progress in Math., 81 (1990), 155-200.

[71] G. Gras, J-F. Jaulent, Sur les corps de nombres réguliers, Math. Z., 202(3) (1989), 343-365.

[72] J-F. Jaulent, T. Nguyen Quang Do, Corps p-rationnels, corps p-réguliers et ramification restreinte, J. Théor. Nombres Bordeaux, 5 (1993), 343-363.

[73] J-F. Jaulent, Classes logarithmiques des corps de nombres, J. Théor. Nombres Bordeaux, 6 (1994), 301-325.

[74] J-F. Jaulent, Classes logarithmiques des corps totalement réels, Acta Arithmetica, 103 (2002), 1-7.

[75] L. J. Federer, B. H. Gross, (with an appendix by W. Sinnott), Regulators and Iwasawa Modules, Invent. Math., 62(3) (1981), 443-457. 
[76] L. V. Kuz'min, The Tate module of algebraic number fields, Izv. Akad. Nauk SSSR, 36 (1972), $267-327$.

[77] J-F. Jaulent, Sur les conjectures de Leopoldt et de Gross, Actes des Journées Arithmétiques de Besançon (1985), Astérisque, 147/148 (1987), 107-120.

[78] J-F. Jaulent, Sur les normes cyclotomiques et les conjectures de Leopoldt et de Gross-Kuz'min, Annales. Math. Québec, 41 (2017), 119-140.

[79] J-F. Jaulent, Classes logarithmiques et capitulation, Functiones et Approximatio, 54(2) (2016), 227-239.

[80] J-F. Jaulent, Sur la capitulation pour le module de Bertrandias-Payan, Pub. Math. Besançon (Théorie des Nombres) (2016), 45-58.

[81] J-F. Jaulent, Principalisation abélienne des groupes de classes de rayons (2018), (in press).

[82] J-F. Jaulent, Principalisation abélienne des groupes de classes logarithmiques, Funct. Approx. Comment. Math., 61(2) (2019), 257-275.

[83] R. I. Berger, G. Gras, Regular fields: normic criteria in p-extensions, Pub. Math. Besançon (Théorie des Nombres), Années 1991/92.

[84] T. Nguyen Quang Do, Lois de réciprocité primitives, Manuscripta Math., 72(1) (1991), 307-324.

[85] J-F. Jaulent, La théorie de Kummer et le $\mathrm{K}_{2}$ des corps de nombres, J. Théor. Nombres Bordeaux, 2 (1990), $377-411$.

[86] K. Belabas, J-F. Jaulent, The logarithmic class group package in PARI/GP, Pub. Math. Besançon (Théorie des Nombres) (2016), 5-18.

[87] T. Nguyen Quang Do, Analogues supérieurs du noyau sauvage, J. Théor. Nombres Bordeaux, 2(4) (1991), $263-271$.

[88] J-F. Jaulent, A. Michel, Approche logarithmique des noyaux étales des corps de nombres, J. Number Theory, 120 (2006), $72-91$

[89] K. Hutchinson, Tate kernels, étale K-theory and the Gross kernel, (2017), arxiv:1709.06465 [math.NT].

[90] F. Hajir, C. Maire, R. Ramakrishna, Cutting towers of number fields, (2019), arxiv:1901.04354 [math.NT].

[91] F. Hajir, C. Maire, R. Ramakrishna, Infinite class field towers of number fields of prime power discriminant, (2019), arxiv:1904.07062 [math.NT].

[92] A. Angelakis, P. Stevenhagen, Absolute abelian Galois groups of imaginary quadratic fields, In: proceedings volume of ANTS-X, UC San Diego 2012, OBS 1 (2013).

[93] A. Angelakis, Universal Adelic Groups for Imaginary Quadratic Number Fields and Elliptic Curves, Thesis University Bordeaux I and University Leiden, 2015.

[94] M. Onabe, On the isomorphisms of the Galois groups of the maximal abelian extensions of imaginary quadratic fields, Natur. Sci. Rep. Ochanomizu Univ., 27(2) (1976), 155-161.

[95] G. Gras, On the structure of the Galois group of the Abelian closure of a number field, J. Théor. Nombres Bordeaux, 26(3) (2014), 635-654.

[96] G. Gras, Les $\theta$-régulateurs locaux d'un nombre algébrique : Conjectures p-adiques, Canadian J. Math., 68(3) (2016), 571-624.

[97] E. Z. Goren, Hasse invariants for Hilbert modular varieties, Isr. J. Math., 122 (2001), 157-174.

[98] G. Boeckle, D.-A. Guiraud, S. Kalyanswamy, C. Khare, Wieferich Primes and a mod p Leopoldt Conjecture, (2018), arxiv:1805.00131 [math.NT].

[99] R. Greenberg, On the Iwasawa invariants of totally real number fields, Amer. J. Math., 98(1) (1976), $263-284$.

[100] T. Nguyen Quang Do, Formules de genres et conjecture de Greenberg, Ann. Math. Québec, 42(2) (2018), 267-280.

[101] G. Gras, Approche p-adique de la conjecture de Greenberg pour les corps totalement réels, Ann. Math. Blaise Pascal, 24(2) (2017), 235-291.

[102] G. Gras, Normes d'idéaux dans la tour cyclotomique et conjecture de Greenberg, Ann. Math. Québec, Online: 17 October 2018, 1-32.

[103] J-F. Jaulent, Normes universelles et conjecture de Greenberg, (2019), arxiv:1904.07014 [math.NT].

[104] J-F. Jaulent, Note sur la conjecture de Greenberg, J. Ramanujan Math. Soc., 34(1) (2019), 59-80.

[105] G. Gras, Principalisation d'idéaux par extensions absolument abéliennes, J. Number Theory, 62(2) (1997), 403-421. 
[106] S. Bosca, Principalization of ideals in abelian extensions of number fields, Int. J. Number Theory, 5(03) (2009), 527-539.

[107] T. Nguyen Quang Do, Sur la conjecture faible de Greenberg dans le cas abélien p-décomposé, Int. J. Number Theory, 2 (2006), 49-64.

[108] T. Nguyen Quang Do, Sur une forme faible de la conjecture de Greenberg II, Int. J. Number Theory, 13 (2017), 1061-1070.

[109] R. Greenberg, Galois representations with open image, Ann. Math. Québec, special volume in honor of Glenn Stevens, 40(1) (2016), 83-119.

[110] F. Pitoun, Calculs théoriques et explicites en théorie d'Iwasawa, Thèse de doctorat en Mathématiques, Laboratoire de Mathématiques, Université de Franche-comté Besançon, 2010.

[111] F. Pitoun, F. Varescon, Computing the torsion of the p-ramified module of a number field, Math. Comp., 84(291) (2015), $371-383$.

[112] J. H. Silverman, Wieferich's criterion and the abc-conjecture, J. Number Theory, 30 (1988), $226-237$.

[113] H. Graves, M. R. Murty, The abc conjecture and non-Wieferich primes in arithmetic progressions, J. Number Theory, 133(6) (2013), 1809-1813.

[114] C. Maire, M. Rougnant, A note on p-rational fields and the abc-conjecture (2019), Proceedings of the A.M.S., (to appear), arxiv:1903.11271 [math.NT].

[115] D. Byeon, Indivisibility of special values of Dedekind zeta functions of real quadratic fields, Acta Arithmetica, 109(3) (2003), 231-235.

[116] J. Assim, Z. Bouazzaoui, Half-integral weight modular forms and real quadratic p-rational fields, (2018/2019), arxiv:1906.03344 [math.NT].

[117] J. Shu, Root numbers and Selmer groups for the Jacobian varieties of Fermat curves, (2018), arxiv:1809.09285 [math.NT].

[118] R. Davis, R. Pries, Cohomology groups of Fermat curves via ray class fields of cyclotomic fields, (2018), (in press).

[119] A. Charifi, Groupes de torsion attachés aux extensions Abéliennes p-ramifiées maximales (cas des corps totalement réels et des corps quadratiques imaginaires), Thèse de $3^{e}$ cycle, Mathématiques, Université de Franche-Comté, 1982,50 pp.

[120] K. Hatada, Mod 1 distribution of Fermat and Fibonacci quotients and values of zeta functions at $2-p$, Comment. Math. Univ. St. Pauli, 36 (1987), 41-51.

[121] K. Hatada, Chi-square tests for mod 1 distribution of Fermat and Fibonacci quotients, Sci. Rep. Fac. Educ., Gifu Univ., Nat. Sci., 12 (1988), 1-2.

[122] T. Hofmann, Y. Zhang, Valuations of p-adic regulators of cyclic cubic fields, J. Number Theory, 169 (2016), 86-102.

[123] J.S. Kraft, R. Schoof, Computing Iwasawa modules of real quadratic number fields, Compositio Math., 97 (1995), $135-155$.

[124] R. Barbulescu, J. Ray, Some remarks and experimentations on Greenberg's p-rationality conjecture, (2017), arxiv:1706.04847 [math.NT].

[125] Z. Bouazzaoui, Fibonacci sequences and real quadratic p-rational fields, (2019), arxiv:1902.04795 [math.NT].

[126] M. Ozaki, H. Taya, A note on Greenberg's conjecture for real abelian number fields, Manuscripta Math., 88(1) (1995), 311-320.

[127] C. Maire, M. Rougnant, Composantes isotypiques de pro-p-extensions de corps de nombres et p-rationalité, Publ. Math. Debrecen, 94(1/2) (2019), 123-155.

[128] G. Gras, Invariant generalized ideal classes-Structure theorems for p-class groups in p-extensions, Proc. Indian Acad. Sci. (Math. Sci.), 127(1) ( 2017), 1-34. 